Optimal Procurement of a Credence Good Under Limited Liability

Helmut Bester

Ouyang Yaofu

School of Business \& Economics

Discussion Paper

Economics

$2018 / 1$ 


\title{
Optimal Procurement of a Credence Good UNDER LIMITED LIABILITY*
}

\author{
Helmut Bester ${ }^{\dagger}$ and Ouyang Yaofu ${ }^{\ddagger}$
}

\author{
September 2017
}

\begin{abstract}
This paper analyzes the optimal contract for a consumer to procure a credence good from an expert when (i) the expert might misrepresent his private information about the consumer's need, (ii) the expert might not choose the requested service since his choice of treatment is non-observable, and (iii) limited liability of the expert precludes imposing penalty payments on him. We characterize payments under the optimal contract and show that, compared with the first-best, these induce inefficient undertreatment. We further show that separating diagnosis and treatment increases consumer surplus. Whether it decreases or increases the likelihood of undertreatment, however, depends on the accuracy of the expert's information.

Keywords: credence goods, non-observable treatments, hidden information, moral hazard, limited liability.

JEL Classification No.: D82, D83, D86, I11,
\end{abstract}

\footnotetext{
*We wish to thank Boris Brekhov, Matthias Dahm, Matthias Lang, and the participants of the Berlin Micro Workshop for helpful comments. Helmut Bester acknowledges support by the Berlin Centre for Consumer Policies (BCCP). Ouyang Yaofu acknowledges support by the China Scholarship Council (CSC).

†Freie Universität Berlin, School of Business and Economics, Berlin (Germany); email: hbester@zedat.fu-berlin.de.

tUniversity of International Business and Economics, Department of Economics, Beijing (China); email: yaofuouyang@gmail.com .
} 


\section{Introduction}

This paper analyzes the optimal contract for a consumer to procure a credence good from an expert. There are two incentive problems: First, only the expert privately receives information about the consumer's need by a costless diagnosis. Thus the contract should provide the expert with incentives for truthful reporting. Second, there is a moral hazard problem because the expert's choice of treatment is not observable. Therefore, the contract should induce the expert to perform the treatment as required. In addition, the expert is protected by limited liability that all contractual payments by the consumer must be nonnegative. This implies that the expert cannot be punished too harshly for failure of treatment.

Consider a consumer who needs one of two treatments to solve his problem. When the problem is a minor one, a low-cost treatment is appropriate and sufficient while a high-cost treatment induces overtreatment; when the problem is a major one, the high-cost treatment is proper and necessary while the low-cost treatment induces undertreatment as it fails. The consumer only knows the prior probability of his problem, while the expert privately observes an informative but noisy signal about the consumer's need. The consumer offers a contract to the expert before the expert observes the signal; he may choose to implement either the high-cost treatment or the low-cost treatment based on his prior information only. Alternatively, he may implement a treatment relying on the expert's information. While the credence goods literature mostly does not use a contract-theoretic approach, we adopt a principal-agent approach to characterize the optimal procurement contract. We show that compared with the first-best outcome the optimal contract leads to too much undertreatment, either by too often implementing the low-cost treatment or by too much reliance on the expert's noisy signal. Furthermore, inefficient undertreatment, although possibly being mitigated, still persists when diagnosis and treatment are separately provided by two experts.

Our analysis considers a typical credence goods environment where a consumer wants to buy a service from an expert who knows better the consumer's need than the consumer himself $]^{1}$ The expert, who is the seller of the service, might misrepresent his superior information in order to exploit the consumer. This happens because even if the consumer can determine whether his problem is solved or not, he does not know which service he really needs. Furthermore, the consumer might also be unable to determine the type of service he received. Therefore, the expert might even charge a price for an unperformed service and remain undetected. Due to the expert's opportunistic behavior, three types of inefficiencies can arise: ${ }^{2}$ first, undertreatment arises when the expert provides an insufficient low-cost

\footnotetext{
${ }^{1}$ The notion of credence goods was first introduced by Darby and Karni (1973). Differing from search goods and experience goods (Nelson, 1970), the consumer of a credence good not only ex ante does not know his need, but even ex post he cannot detect whether his need is properly satisfied. Typical credence goods include education, repair service, taxi service and medical service. The notion of credence goods has also been applied to many other goods that bear the "credence property", such as patents (Thambisetty, 2007), contracting for infrastructure projects (Dulleck, Gong, and Li, 2015).

${ }^{2}$ In addition, a forth inefficiency, duplication of search and diagnosis cost, might arise when it is possible for the consumer to search for second opinions. See Wolinsky (1993) and Pesendorfer and Wolinsky (2003).
} 
treatment which does not solve the consumer's problem; second, overtreatment arises when the expert provides an unnecessary high-cost treatment as a low-cost treatment would have been sufficient; third, overcharging arises when the expert charges the price of a service he did not perform. $3^{3}$

Ample evidence shows that inefficiencies are prominent in credence goods markets. For example, Schneider (2012, p. 3) undertakes a field experiment in the car-repair service market, and finds that both under- and overtreatment are pervasive: "mechanics recommended completely unnecessary repairs in 33 percent of the experimental visits and 27 percent of the Canadian visits ... seventy-five percent of the field experiment visits were characterized by serious undertreatment." Similar results have been found by a natural field experiment on taxi rides in Greece (Balafoutas, Beck, Kerschbamer, and Sutter, 2013). The taxi drivers not only overtreated the uninformed passengers by taking them on significantly longer detours, but also overcharged those who lacked information on the local tariff system by about $5 \%$. More significantly, overtreatment has been listed as one of six major categories for waste in the US health care spending by Berwick and Hackbarth (2012, p. 1514). They estimated that "this category represented between $\$ 158$ billion and $\$ 226$ billion in wasteful spending in 2011". In a similar vein, a recent OECD report states: "Examples of unnecessary or inappropriate care abound at all points of the care pathway, starting with overtesting and overdiagnosis. Unnecessary use of surgical procedures is not an exception. ... Excessive use of medicines is also an issue; for instance, half of antimicrobial prescriptions are inappropriate.' 4

Our analysis shows that some inefficiencies are in fact unavoidable even in the optimal contract. Not only the choice of treatment may be distorted by asymmetric information, but also the payments for a treatment may not reflect its cost. We show that it is optimal to make payments more contingent on success and failure of a treatment rather than its cost. This may imply that necessarily some kind of overcharging occurs: after a successful treatment the expert may be entitled to a payment which is higher than the cost of a high-cost treatment even when he performs only a low-cost treatment.

Dulleck and Kerschbamer (2006) survey the credence goods literature within a unified framework. They show that the first-best can be implemented when either the expert is liable for the resolution of the consumer's problem (Liability), or the type of treatment provided by the expert is verifiable and contractible (Verifiability). When neither liability nor verifiability are satisfied, however, the credence goods market becomes a "market for lemons" (Akerlof, 1970): the quality of service deteriorates, or the market breaks down all together.

We contribute to this literature in three ways. First, we depart from much of the credence goods literature by employing a principal-agent contracting approach, which is adequate to analyze problems of asymmetric information in contracting. Second, we assume neither liability nor verifiability. As will be shown below, large parts of the credence goods literature

\footnotetext{
${ }^{3}$ Although overcharging is a pure transfer between the expert and the consumer, it can also cause social inefficiency by deterring some consumers from demanding the service or a dead-weight loss of taxation if partially publicly funded.

${ }^{4}$ OECD (2017, p. 51), Tackling Wasteful Spending on Health, OECD Publishing, Paris. http://dx.doi.org/10.1787/9789264266414-en.
} 
assumes either liability or verifiability or both in their analysis. These studies are relevant in some situations, but have limited applicability in other situations where either verifiability or liability is not likely to hold. These situations are not uncommon in credence goods markets. In the case of a medical treatment, for instance, the patient may be unable to observe the treatment performed by the expert, while the physician is not liable for the outcome of treatment. Third, we follow Bester and Dahm (2017) by assuming that the expert's diagnosing technology is not perfectly precise. This implies that implementing treatments based on the expert's signal is not always optimal. Even if the expert reports truthfully, the signal might be incorrect, and thus both under- and overtreatment might occur. Furthermore, we also generalize the expert's treatment technology to be not perfectly effective: even a proper treatment fails with small but positive probability. This generalization is not only realistic, but also necessary for non-observability of the expert's choice of treatment. This is so because otherwise one could infer that the expert did not perform the high-cost treatment if the treatment fails.

In our model success and failure of treatment are observable and contractible. We first study whether the consumer can design contingent payments in such a way that the expert adopts one of three possible treatment strategies: the high-cost treatment $(\mathbf{H})$ or the low-cost treatment (L) independently of the diagnosis outcome, or a treatment choice (S) contingent on the signal observed by the expert after signing the contract. By the Revelation Principle (Myerson, 1979) and its extended version (Myerson, 1982), there is no loss of generality in considering only incentive compatible contracts under which the expert will not only report truthfully but also implement the treatment as required. In a first step we characterize the optimal payments for implementing each of the three treatment strategies. We then derive the overall optimal contract, which maximizes the consumer's surplus over the three treatment strategies.

Our main result shows that all three treatment strategies are implementable by properly designed contracts. Also, each of them can be optimal for some values of the consumer's prior. When the prior is highly informative, it is optimal for the consumer to implement either $\mathbf{H}$ or $\mathbf{L}$ by not making use of the expert's information. But when the prior is not informative enough, utilizing the expert's signal by implementing strategy $\mathbf{S}$ is optimal. Compared with the first-best outcome, however, the overall optimal contract leads to too much undertreatment in two ways: (i) by implementing too often the low-cost strategy L when the consumer's problem is more likely minor; (ii) by implementing too often strategy $\mathbf{S}$ when the consumer's problem is more likely major. The driving force behind this result is basically a tradeoff between rent extraction and efficiency.

Intuitively, since the expert is protected by limited liability and his choice of treatment is unobservable, implementing a high-cost treatment under $\mathbf{H}$ or, with positive probability, under $\mathbf{S}$ must leave a positive rent to the expert. When the consumer's problem is more likely minor, strategy $\mathbf{H}$ is more likely not needed. The consumer then chooses either to implement treatment strategy $\mathbf{L}$ or $\mathbf{S}$. Due to the rent involved in the latter strategy, the overall optimal contract requires implementing more often the low-cost treatment only to avoid leaving such a rent. When the consumer's problem is more likely major, a low-cost treatment is more likely insufficient. The consumer then chooses either to implement treatment $\mathbf{H}$ 
or $\mathbf{S}$. Interestingly, although implementing treatment $\mathbf{S}$ requires an incentive constraint for truthful reporting, which is not required for $\mathbf{H}$, the expert's rent under $\mathbf{S}$ is comparatively smaller. Therefore, the overall optimal contract involves too much reliance on the expert's information by implementing $\mathbf{S}$.

In Section 6 we extend our model to the case where diagnosis and treatment are separately provided by two experts, and study whether such an arrangement can avoid the efficiency losses identified above. Separating diagnosis and treatment is feasible in some situations. For example, diagnosing and prescribing can be separated from selling drugs. Indeed, such a practice has been adopted by many countries in their health care systems such as Germany and the UK. In other situations, nevertheless, this separation is almost impossible..$^{5}$ We find that separating diagnosis and treatment reduces some, but not all, of the inefficiencies when the consumer's problem is more likely minor or the expert's signal is highly accurate. Otherwise, however, separation even increases the likelihood of inefficient undertreatment. Intuitively, separating the two procedures solves the adverse selection problem related to the expert's report. This decreases the limited liability rent for the expert under treatment strategies $\mathbf{H}$ and $\mathbf{S}$. Therefore, when the consumer's problem is more likely minor, separation reduces inefficient undertreatment as implementing treatment strategy $\mathbf{S}$ involves less rent for the expert. But when the consumer's problem is more likely major, separation can either decrease or increase the rent difference between implementing treatment strategies $\mathbf{H}$ and $\mathbf{S}$. This depends on the accuracy of the expert's information: when it is sufficiently precise, separation reduces the rent difference and the inefficiency of undertreatment under S. Otherwise, the difference is increased and so inefficient undertreatment becomes more likely as strategy $\mathbf{S}$ becomes optimal. For the consumer, however, separation is beneficial irrespective of the efficiency effects. He can appropriate a larger share of the surplus under strategies $\mathbf{H}$ and $\mathbf{S}$ because separation mitigates the adverse selection problem of truthfully revealing the information of the diagnosis.

\section{Related Literature}

Most of the literature on credence goods assumes either that the expert is liable for the resolution of the consumer's problem (Liability), or that the type of treatment provided by the expert is verifiable and contractible (Verifiability), or both. The typical results are as follows: (1) when verifiability holds, overcharging can be avoided; ${ }^{6}$ (2) when liability holds, undertreatment can be precluded:77 (3) when both verifiability and liability hold, both overcharging and undertreatment can be prevented. $]^{8}$ The relevance of these three cases depends on the market environment. Verifiability is more plausible when no special expertise

\footnotetext{
${ }^{5}$ See more examples and further discussion for the feasibility of the separation in Darby and Karni (1973), Emons (1997), Dulleck and Kerschbamer (2006), and Bester and Dahm (2017).

${ }^{6}$ See Emons (1997),Emons (2001). Pesendorfer and Wolinsky (2003), Dulleck and Kerschbamer (2009), Bonroy, Lemarié, and Tropéano (2013), Fong, Liu, and Wright (2014), Hilger (2016)

'See Pitchik and Schotter (1987), Wolinsky (1993), Emons (2001). Fong (2005), Sülzle and Wambach (2005). Hyndman and Ozerturk (2011), Gabszewicz and Resende (2012) Liu (2011).

${ }^{8}$ See Taylor (1995), Alger and Salanié (2006), Dulleck and Kerschbamer (2009).
} 
is needed for verifying the type of treatment, such as in the taxi-cab service. Liability is more likely to hold when the outcome of treatment is easily observable and verifiable. For instance, in the taxi-cab service the consumer can easily tell whether his destination is reached or not. ${ }^{9}$ But also situations where neither verifiability nor liability holds are not unrealistic in credence goods markets. Our paper addresses this kind of environment, which is largely neglected in the literature 10

There are only a few papers which like us employ a contracting approach to the credence goods problem. Demski and Sappington (1987) study optimal contracts between a principal and an agent to induce the agent to acquire costly expertise. This corresponds to the moral hazard problem of costly diagnosis in the context of credence goods. To focus on the delegated expertise problem, they further assume treatments to be costless so that the choice of treatment does not involve a problem of moral hazard. They also preclude any communication between the expert and the principal.

Dulleck, Gong, and $\mathrm{Li}$ (2015) model contracting for an infrastructure project as a credence good. They analyze how a procurer can motivate contractors to invest in costly design efforts and to reveal the proper design. While our paper provides conditions under which the consumer will make use of the expert's information, in their paper the procurer has to rely on the contractors' information, because an unguided guess will never yield the right choice. Also in their model it is impossible to condition the contract upon the outcome of the project, whereas we consider payments contingent on success or failure.

Most closely related to our analysis is the contracting model of Bester and Dahm (2017), who like us consider an expert whose diagnosis technology is noisy. But they focus on a two-sided incentive problem: investing in costly diagnosis on the expert's side and subjective evaluation of success and failure of the treatment on the consumer's side. There are two key modeling differences. First, they assume that the expert's choice of treatment is observable and verifiable. So, the moral hazard problem related to the expert's choice of treatment considered in our model does not exist in their model. Second, in their paper the expert is liable for the resolution of the consumer's problem as he has to implement the high-cost treatment following a failure reported in the first-period. In contrast, our paper focuses on the tradeoff between rent extraction under limited liability and efficiency. Bester and Dahm (2017) also consider the effects of separating diagnosis and treatment. They show that the first-best outcome is always implementable if separation is possible. This is not true in our setting. Indeed, we show that separation may even increase the likelihood of undertreatment for some parameter combinations.

This paper is organized as follows. The next section presents the model with our assumptions on observability and contractability. In Section 3 we characterize the first-best treatment strategies as a reference point. Section 4 studies the optimal contract for implementing a given treatment strategy. In Section 5 we derive the overall optimal contract

\footnotetext{
${ }^{9}$ Dulleck and Kerschbamer (2006) offer detailed discussions about verifiability and liability in repair service, taxi cab, and medical treatment markets .

${ }^{10} \mathrm{An}$ exception is Emons (2001). In his model a monopolistic expert chooses a capacity, which can be allocated between diagnosis and repair. As the consumer cannot observe the expert's service, the expert has strong incentives to cheat on services. The market may even break down because the consumer refrains from consulting the expert.
} 


\begin{tabular}{|c|cc|}
\hline & $\theta_{L}$ & $\theta_{H}$ \\
\hline$T_{L}$ & $\rho$ & 0 \\
$T_{H}$ & $\rho$ & $\rho$ \\
\hline
\end{tabular}

Table 1: Success Probabilities

for the consumer and establish our main results. Section 6 extends our model to situations where diagnosis and treatment can be separated. Finally, we conclude in Section 7. Some lengthier proofs are relegated to the Appendix.

\section{The Model}

Consider a credence good problem with one consumer and one expert. The consumer has a problem $\theta$ which is either a minor one, $\theta_{L}$, or a major one, $\theta_{H}$. If the problem is solved, he gets zero utility. Otherwise, he suffers a loss of $-L$, with $L>0$.

The expert can provide two types of treatment, $T_{H}$ and $T_{L}$. Abusing notation we assume that the expert's cost of performing treatment $T_{H}$ is also $T_{H}>0$ while the cost of treatment $T_{L}$ is $T_{L}$, which is normalized to zero. Also, the expert's outside option is normalized to zero. Treatment $T_{H}$ solves both types of problem with probability $0<\rho<1$. Treatment $T_{L}$ solves problem $\theta_{L}$ with probability $\rho$ and problem $\theta_{H}$ with probability zero. The success probabilities of the combinations of treatment and problem are illustrated in Table 1 .

Our assumption on the effectiveness of expert's treatment modifies the standard assumption in the literature on credence goods that $\rho=111$ We obtain the standard assumption as the limit $\rho \rightarrow 1$. As we will assume that the expert's choice of treatment is not observable, the assumption $\rho<1$ also ensures that failure of treatment does not reveal that the expert did not perform treatment $T_{H}$. In fact, it makes sense that the success of treatment cannot always be guaranteed. The outcome may depend not only on the type of treatment but also on other unpredictable contingencies, which potentially lead to failure. In the medical service, for instance, the proposed treatment by a doctor, even if it is indeed appropriate and standard based on medical knowledge existing at the relevant time, may fail in some situations. ${ }^{12}$ In addition, the results can vary from case to case since medicine is not an exact science 13

\footnotetext{
${ }^{11}$ See the survey by Dulleck and Kerschbamer (2006). There are two exceptions. Glazer and McGuire (1996) assume that there are two types of expert: a safe type (S) and a risky type (R). Type $\mathrm{S}$ can solve the problem for sure, while the R-type only repairs it with some probability depending on the consumer's type. Richardson (1999) assumes, like us, that treatment is only effective with probability smaller than one. But there is only one treatment available in his setting.

${ }^{12}$ Situations that involve the risk of failure are not only well recognized, but they should be disclosed to the consumer within the consent form according to the medical practice in some countries. See.e.g, 12 key points on consent: the law in England, published by the Department of Health of the UK, March 2001.

${ }^{13}$ This point has been recognized in Dulleck and Kerschbamer (2006, p. 32) where they write "...With many sicknesses there is no sufficient treatment, with others success is only random. Thus, a failing treatment is no perfect signal of undertreatment...". In addition, this is also the reason for why a medical doctor is not fully liable for the outcome of treatment.
} 
To ensure that a positive net expected surplus can be achieved by performing the appropriate treatment, we assume that

$$
\rho L>T_{H}>T_{L}=0 \text {. }
$$

We speak of undertreatment when the major problem $\theta_{H}$ is treated with the low-cost treatment $T_{L}$ as it is totally unhelpful and always fails. While the minor problem is solved by the high-cost treatment in the combination $\left(\theta_{L}, T_{H}\right)$, we speak of overtreatment as the low-cost treatment $T_{L}$ would be as effective as the high-cost treatment $T_{H}$.

Information and Observability The consumer knows that he has a problem but is uncertain as to which type it is. He knows, however, the prior probability

$$
\operatorname{Prob}\left(\theta_{L}\right)=1-\operatorname{Prob}\left(\theta_{H}\right)=q
$$

The expert not only knows the prior in (2), but also by performing a costless diagnosis he can privately observe a noisy signal $s_{i} \in\left\{s_{L}, s_{H}\right\},{ }^{14}$ The signal is correct with probability $\sigma \in(1 / 2,1)$, i.e.

$$
\operatorname{Prob}\left(s_{L} \mid \theta_{L}\right)=\operatorname{Prob}\left(s_{H} \mid \theta_{H}\right)=\sigma, \quad \operatorname{Prob}\left(s_{L} \mid \theta_{H}\right)=\operatorname{Prob}\left(s_{H} \mid \theta_{L}\right)=1-\sigma
$$

This assumption generalizes the usual assumption $\sigma=1$ in most of literature on credence goods ${ }^{15}$ After observing the signal, the expert can be obliged to report a signal to the consumer. As the signal is the expert's private information, this creates an adverse selection problem. The expert might behave opportunistically by reporting a signal which he actually did not observe.

We assume that success or failure of treatment is publicly observable, but the expert's choice of treatment is unobservable. Therefore, the payments for the expert can be contingent on the outcome of treatment, but not directly on the type of treatment. This is likely to hold when identifying the treatment requires specific expertise but checking the outcome of treatment needs few experience or skills. Take the car-repair service as an example. It is obvious whether the car works or not after repair but identifying the type of repair may require further knowledge. The non-observability of the expert's choice of treatment gives rise to an additional problem of moral hazard in our model. The expert might provide treatment $T_{L}$, instead of treatment $T_{H}$ as required, to save some treatment cost.

Contracts The consumer can decide to design a contract that implements either treatment $T_{L}$ or $T_{H}$ based on his prior information only. Or he can decide to implement treatment $T_{i}$ when the expert observes a signal $s_{i}$ with $i \in\{H, L\}$. Indeed, the consumer prefers to follow

\footnotetext{
${ }^{14}$ The costless diagnosis assumption is appropriate when the diagnosis cost is negligible compared to the treatment cost, or alternatively the diagnosis is a by-product of the provision of the treatment. This assumption is also common in this literature, e.g, Pitchik and Schotter (1987), Fong (2005), Liu (2011), Fong, Liu, and Wright (2014), Hilger (2016).

${ }^{15}$ An exception is Bester and Dahm (2017). But in their setup diagnosing is costly for the expert and diagnosis effort is not observable. This creates a moral hazard problem of information acquisition, which we do not consider in our model.
} 
his prior information if it is informative enough to identify the proper treatment. Otherwise, he better trusts the expert's signal and implements the treatment according to the expert's information. Therefore, a contract can specify one of the following three treatment strategies:

- Treatment strategy $\mathbf{L}$ : the expert provides treatment $T_{L}$. The payment for the expert $p_{L} \in\left(p_{L s}, p_{L f}\right)$ is designed such that the expert will indeed always implement treatment $T_{L}$, where the payment $p_{L s}$ is paid if the treatment succeeds while $p_{L f}$ is paid otherwise.

- Treatment strategy $\mathbf{H}$ : the expert provides treatment $T_{H}$. Likewise, the payment for the expert $p_{H} \in\left(p_{H s}, p_{H f}\right)$ is designed such that the expert will indeed always implement treatment $T_{H}$, where the payment $p_{H s}$ is paid if the treatment succeeds while $p_{H f}$ is paid otherwise.

- Treatment strategy $\mathbf{S}$ : the expert reports to the consumer a signal $\hat{s}_{i} \in\left\{s_{L}, s_{H}\right\}$ and then implements treatment $T_{i}$ upon reporting signal $\hat{s}_{i}$ for $i \in\{H, L\}$. The payment for the expert is designed such that the expert will truthfully report a signal $\hat{s}_{i}=s_{i}$ and at the same time honestly implement treatment $T_{i}$ upon observing a signal $s_{i}$ for $i \in\{H, L\}$. Specifically, the payment scheme is $\left\{p_{L}, p_{H}\right\} \equiv\left\{\left(p_{L s}, p_{L f}\right),\left(p_{H s}, p_{H f}\right)\right\}$. When the expert reports a signal $\hat{s}_{i}$ with $i \in\{H, L\}$, he receives the payment $p_{i s}$ if the treatment succeeds and $p_{i f}$ otherwise.

Note that both strategies $\mathbf{H}$ and $\mathbf{S}$ are subject to the moral hazard problem concerning the expert's choice of treatment as indicated above. Moreover, strategy $\mathbf{S}$ involves in addition the adverse selection problem of the expert's private diagnosis information. We indicate the consumer's contract decision by its treatment strategy $\mathbf{D} \in\{\mathbf{L}, \mathbf{H}, \mathbf{S}\}$.

Timing The contracting relation proceeds in the following sequence:

1. Nature draws the consumer's type $\theta \in\left\{\theta_{L}, \theta_{H}\right\}$. The realization of $\theta$ is unobservable to both the consumer and the expert. Both parties only know the ex ante probabilities as given by (2).

2. The consumer offers a contract $\mathbf{D} \in\{\mathbf{L}, \mathbf{H}, \mathbf{S}\}$ to the expert. This contract stipulates the expert to perform treatment $T \in\left\{T_{L}, T_{H}\right\}$ or to implement treatment $T_{i}$ upon the expert observing a signal $s_{i} \in\left\{s_{H}, s_{L}\right\}$, and also specifies the corresponding payments as stated above. If the expert rejects the contract, the contracting game ends.

3. If the expert accepts the contract offer, he performs a costless diagnosis and privately observes a signal $s_{i} \in\left\{s_{L}, s_{H}\right\}$. Under the contract decision $\mathbf{D} \in\{\mathbf{L}, \mathbf{H}\}$ the expert performs a treatment which can be either the mandated treatment or not. If $\mathbf{D}=\mathbf{S}$, the expert reports a signal $\hat{s}_{i} \in\left\{s_{L}, s_{H}\right\}$ and then performs a treatment $T_{j} \in\left\{T_{H}, T_{L}\right\}$. The expert may report a signal that he did not observe, i.e, $\hat{s}_{i} \neq s_{i}$. He may also choose a treatment $T_{j} \neq T_{i}$ upon observing a signal $s_{i}$, for $i, j \in\{H, L\}$.

4. The success or failure of the treatment is publicly observed. Under a contract decision $\mathbf{D} \in\{\mathbf{L}, \mathbf{H}\}$, the expert receives his payment based only on the outcome of the treatment according to the contract. If $\mathbf{D}=\mathbf{S}$, the expert's payment is based on both his report and the outcome as specified above. 
The contract is offered at the ex ante stage, i.e. before the expert observes the signal. This precludes asymmetric information at the contracting stage, which would allow the expert to extract a rent by his informational advantage. We also assume that the expert is protected by limited liability so that all payments must be nonnegative. This assumption implies that the expert cannot be punished too harshly when the treatment fails.

In stage 2 of the above sequence of events, the contract is proposed as a take-it-or-leave-it offer by the consumer to the expert. This maximizes the consumer's surplus subject to the informational constraints of adverse selection and moral hazard, and the institution of limited liability. But the outcome of this bargaining game can also be obtained as the equilibrium of Bertrand competition in a market where two or more identical experts compete for consumers by offering contracts for their diagnosis and treatment service. Competition then forces the experts to maximize the consumers' surplus, subject to the same incentive restrictions and limited liability constraints as in our subsequent analysis.

\section{First-Best Treatment Strategy}

Before analyzing the optimal contract between the consumer and the expert, we first derive as a benchmark the first-best outcome in the absence of asymmetric information and moral hazard $\sqrt{16}$ Suppose that the expert's signal is publicly observable and the treatment action is also contractible. This is plausible when the consumer himself is also an expert, for instance, when a car-mechanic repairs his own car. In this situation, there exists no incentive problem and thus no need to condition the expert's payment on the outcome of treatment, in particular, rewarding success while punishing failure ${ }^{17}$ The limited liability assumption is trivially satisfied because otherwise the expert would reject the contract. The consumer's problem then is reduced to maximizing the overall surplus subject to the expert's participation constraint only, which results in the first-best outcome.

As explained above, the consumer has the three possible treatment strategies $\mathbf{D} \in\{\mathbf{L}, \mathbf{H}, \mathbf{S}\}$ at the contracting stage. First, under $\mathbf{L}$ the consumer chooses to contract for treatment $T_{L}$. He optimally offers a take-it-or-leave-it contract at a price $p_{L}=T_{L}=0$, which is the lowest acceptable price for the expert. Under this contract the consumer's net surplus is

$$
S_{L}^{*} \equiv \rho q L-T_{L}=\rho q L,
$$

because treatment $T_{L}$ solves problem $\theta_{L}$ with probability $\rho$ but fails for problem $\theta_{H}$. Thus, undertreatment occurs with probability $(1-q)$.

\footnotetext{
${ }^{16}$ This analysis is closely related to the characterization of efficient polices for the first-best outcome in Dulleck and Kerschbamer (2009) and Bester and Dahm (2017). But there are two key differences. First, we consider a more general setting where even a proper treatment might fail with small but positive probability. Second, both papers assume that the expert is liable for the resolution of the consumer's problem: a second-period high-cost treatment must be implemented whenever the first-period one fails.

${ }^{17}$ This argument holds because both the consumer and the expert are risk-neutral. In other cases, the payments for success and failure might still differ as a way to transfer risk between the consumer and the expert.
} 
Second, the consumer can also implement $\mathbf{H}$ by offering a take-it-or-leave-it contract at a price $p_{H}=T_{H}$. This contract gives the consumer the net surplus

$$
S_{H}^{*}=\rho L-T_{H}
$$

because treatment $T_{H}$ solves both types of problem with probability $\rho$. But it induces overtreatment with probability $q$.

Finally, if under $\mathbf{S}$ the consumer decides to use the expert's signal and to implement treatment $T_{i}$ upon signal $s_{i}$, it is optimal to offer a take-it-or-leave-it contract $\left(p_{L}, p_{H}\right)$ with $p_{L}=T_{L}=0$ and $p_{H}=T_{H}$. Under this contract, the consumer pays $p_{L}$ for treatment $T_{L}$ after observing a signal $s_{L}$, and $p_{H}$ for treatment $T_{H}$ after a signal $s_{H}$. The consumer's expected surplus from this treatment strategy is

$$
S_{S}^{*}=q \sigma \rho L+[q(1-\sigma)+(1-q) \sigma]\left(\rho L-T_{H}\right) .
$$

The first term on the right-hand-side in (6) relates to the case where the expert's signal is $s_{L}$, which happens with probability $[q \sigma+(1-q)(1-\sigma)]$. In this case treatment $T_{L}$ with zero cost is implemented and repairs the consumer's problem with probability $q \sigma \rho$. The second term corresponds to the case where the signal is $s_{H}$, which happens with probability $[q(1-\sigma)+(1-q) \sigma]$. In this case treatment $T_{H}$ is implemented, which repairs the consumer's problem with probability $\rho$ at the cost $T_{H}$. Note that undertreatment only occurs when the expert receives an incorrect signal $s_{L}$, while overtreatment arises with an incorrect signal $s_{H}$. A more precise signal, however, can alleviate both under- and overtreatment, and thus is beneficial for the consumer because

$$
\partial S_{S}^{*} / \partial \sigma=(1-q) \rho L-(1-2 q) T_{H}>0
$$

by assumption (1).

Based on the consumer's surplus from the three treatment strategies in (4)-(6), we can now derive the consumer's first-best treatment strategy. If the treatment is chosen based on the prior only, then implementing treatment $T_{L}$ is at least as good as implementing treatment $T_{H}$ if and only if $S_{L}^{*} \geq S_{H}^{*}$, which is equivalent to

$$
q \geq \bar{q} \equiv 1-\frac{T_{H}}{\rho L}
$$

where $\bar{q} \in(0,1)$ since (1). Intuitively, the more likely the consumer's problem is minor, the larger is the risk of overtreatment by implementing treatment $T_{H}$, while the risk of undertreatment by implementing treatment $T_{L}$ is smaller. Therefore, implementing treatment $T_{L}$ is more desirable when $q$ is larger.

However, implementing the treatment based on the signal is at least as good as implementing treatment $T_{H}$ or $T_{L}$ based on the prior only if and only if $S_{S}^{*} \geq S_{H}^{*}$ and $S_{S}^{*} \geq S_{L}^{*}$, which is equivalent to

$$
q \geq q_{H} \equiv \frac{(1-\sigma)\left(\rho L-T_{H}\right)}{(1-\sigma)\left(\rho L-T_{H}\right)+\sigma T_{H}}
$$

and

$$
q \leq q_{L} \equiv \frac{\sigma\left(\rho L-T_{H}\right)}{\sigma\left(\rho L-T_{H}\right)+(1-\sigma) T_{H}} .
$$




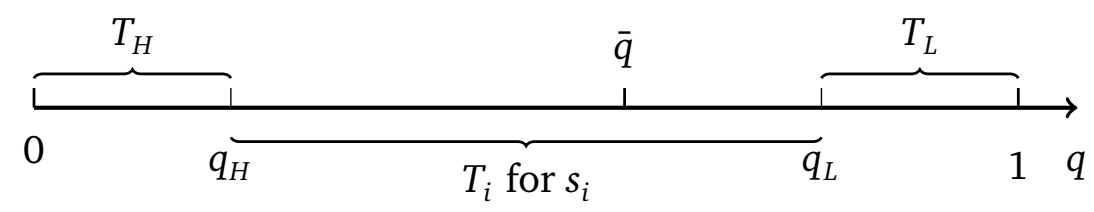

Figure 1: First-best Treatments

Note that $q_{H}<\bar{q}<q_{L}$ as $\sigma \in(1 / 2,1)$. Therefore, the consumer optimally decides to trust the expert's signal and implement the treatment based on the signal if and only if

$$
q_{H} \leq q \leq q_{L}
$$

Note that both $q_{H}$ and $q_{L}$ are a function of the accuracy of the signal $\sigma$ with $\partial q_{H} / \partial \sigma<0$ and $\partial q_{L} / \partial \sigma>0, \lim _{\sigma \rightarrow 1} q_{H}=0$, and $\lim _{\sigma \rightarrow 1} q_{L}=1$.

We summarize the first-best treatment strategy in the following proposition:

Proposition 1. Assume that the expert's signal is publicly observable and his choice of treatment is also contractible. Then the consumer can appropriate the first-best surplus by the following contract:

(a) For $q<q_{H}$, treatment strategy $\boldsymbol{H}$ is optimal with the payment $p_{H}=T_{H}$.

(b) For $q_{H} \leq q \leq q_{L}$, treatment strategy $S$ is optimal with the payment $p_{i}=T_{i}$ for $i \in\{H, L\}$.

(c) For $q>q_{L}$, treatment strategy $L$ is optimal with the payment $p_{L}=T_{L}$.

Figure 1 illustrates Proposition 11. Implementing the treatment based on the signal constitutes the first-best treatment strategy within the interval $\left[q_{H}, q_{L}\right]$. Outside this interval, the high-cost treatment $T_{H}$ is optimal if $q<q_{H}$, and the low-cost treatment $T_{L}$ if $q>q_{L}$.

Intuitively, for $q<q_{H}$ the prior indicates that the problem is more likely major, the risk of overtreatment with implementing treatment $T_{H}$ is comparatively small, while the risk of undertreatment with treatment $T_{L}$ or implementing the treatment based on the signal is comparatively large. Therefore, the first-best requires implementing treatment $T_{H}$ based on the prior only. Vice verse, $q>q_{L}$ implies that the problem is more likely minor, then the risk of overtreatment dominates the risk of undertreatment. Thus, implementing treatment $T_{L}$ based on the prior is the first-best. Furthermore, when the prior becomes perfectly precise as $q \rightarrow 0$ or $q \rightarrow 1$, no further information is needed for deciding proper treatment: implementing treatment $T_{H}$ (for $q=0$ ) or treatment $T_{L}$ (for $q=1$ ) constitutes the first-best. Using the expert's signal, however, becomes preferable for a larger interval of the prior as the signal becomes more precise. Indeed, when the signal becomes perfectly precise in the limit $\sigma \rightarrow 1$, implementing the treatment based on the signal is always the first-best.

\section{Treatment Implementation}

We now analyze the implementation of the three treatment strategies $\mathbf{D} \in\{\mathbf{L}, \mathbf{H}, \mathbf{S}\}$ under the contractual constraints described in Section 2. We successively characterize the optimal 
payments to implement each of the three treatment strategies subject to the incentive constraints of adverse selection (truthful reporting), moral hazard (choice of treatment), and limited liability. This allows us to determine in the next section the overall optimal treatment strategy, which yields the highest consumer surplus among the three strategies.

After the expert privately observes the signal of his diagnosis, the consumer maintains his prior belief in (2) while the expert updates his belief based on the signal. Denote the expert's posterior belief by $\mu_{L}$ and $\mu_{H}$ upon observing signal $s_{L}$ and $s_{H}$, respectively. By Bayes rule, we have

$$
\begin{aligned}
& \mu_{L} \equiv \operatorname{Prob}\left(\theta_{L} \mid s_{L}\right)=1-\operatorname{Prob}\left(\theta_{H} \mid s_{L}\right)=\frac{q \sigma}{q \sigma+(1-q)(1-\sigma)}, \\
& \mu_{H} \equiv \operatorname{Prob}\left(\theta_{L} \mid s_{H}\right)=1-\operatorname{Prob}\left(\theta_{H} \mid s_{H}\right)=\frac{q(1-\sigma)}{q(1-\sigma)+(1-q) \sigma} .
\end{aligned}
$$

Note that

$$
0<\mu_{H}<q<\mu_{L}<1,
$$

since $\sigma \in(1 / 2,1)$. Therefore, the expert and the consumer hold different beliefs about the nature of the consumer's problem after the expert observes the signal.

We begin with the two simple cases in which the consumer wants to implement treatment $T_{L}$ or $T_{H}$ without making use of the expert's diagnosis. In these cases, the expert is not obliged to report a signal to the consumer, but only needs to provide the treatment as required. Therefore, the only incentive problem involved is the moral hazard problem concerning the expert's choice of treatment.

Consider first the case $\mathbf{D}=\mathbf{L}$ where the consumer requires treatment $T_{L}$ from the expert. In this case, there is no moral hazard problem because the expert's cost satisfies $T_{H}>T_{L}=0$. Therefore, to implement $\mathbf{L}$ the consumer can make the payments to the expert independent of the outcome of treatment. If the consumer offers a payment $p_{L}=p_{L s}=p_{L f}$ for treatment $T_{L}$, his expected surplus is

$$
S_{L}=q \rho L-p_{L},
$$

and the expert's profit is

$$
\Pi\left(T_{L}\right)=p_{L}-T_{L}=p_{L} .
$$

Therefore, the expert's participation constraint and the limited liability constraint under the contract are the same:

$$
p_{L} \geq 0
$$

Since obviously the consumer maximizes his expected surplus subject to (IR\&LL) by setting $p_{L}=0$, we have the following result:

Proposition 2. The optimal contract to implement treatment strategy $L$ has the following properties:

(a) The optimal payment is

$$
p_{L}=p_{L s}=p_{L f}=T_{L}=0 .
$$

(b) The expert obtains no rent while the consumer appropriates all economic rents, i.e.

$$
\Pi\left(T_{L}\right)=0, \quad S_{L}=q \rho L .
$$


Because there is no moral hazard problem the consumer appropriates all economic rents and obtains the same surplus as in the first-best.

Next we consider the case $\mathbf{D}=\mathbf{H}$ where the consumer wants to mandate treatment $T_{H}$. In this case there exists a moral hazard problem associated with the expert's choice of treatment. The expert could provide treatment $T_{L}$ rather than $T_{H}$ to reduce his cost. By the Revelation Principle (Myerson, 1979, 1982), we can restrict ourselves to incentive feasible contracts.

Assume that the consumer offers a contract $\left(p_{H s}, p_{H f}\right)$ so that the expert receives the payment $p_{H s}$ if the treatment succeeds, and $p_{H f}$ if it fails. Then, by providing treatment $T_{H}$ as required the expert obtains an expected profit

$$
\Pi\left(T_{H}\right)=\Pi\left(T_{H} \mid s_{L}\right)=\Pi\left(T_{H} \mid s_{H}\right)=\rho p_{H s}+(1-\rho) p_{H f}-T_{H},
$$

as the treatment $T_{H}$ succeeds with probability $\rho$. If, however, the expert selects treatment $T_{L}$ instead, based on his posterior belief in (12) his expected profits after a signal $s_{L}$ and $s_{H}$ are:

$$
\begin{aligned}
& \Pi\left(T_{L} \mid s_{L}\right)=\mu_{L} \rho p_{H s}+\left(1-\mu_{L} \rho\right) p_{H f}-T_{L}, \\
& \Pi\left(T_{L} \mid s_{H}\right)=\mu_{H} \rho p_{H s}+\left(1-\mu_{H} \rho\right) p_{H f}-T_{L},
\end{aligned}
$$

as treatment $T_{L}$ only solves problem $\theta_{L}$ with probability $\rho$.

Therefore, the expert will indeed implement treatment $T_{H}$ upon observing a signal $s_{L}$ and $s_{H}$ if and only if

$$
\Pi\left(T_{H}\right) \geq \Pi\left(T_{L} \mid s_{L}\right), \quad \Pi\left(T_{H}\right) \geq \Pi\left(T_{L} \mid s_{H}\right)
$$

This equivalently implies that the contractual payments must satisfy the following two incentive compatibility constraints:

$$
\begin{aligned}
& \rho\left(1-\mu_{L}\right)\left(p_{H s}-p_{H f}\right) \geq T_{H}, \\
& \rho\left(1-\mu_{H}\right)\left(p_{H s}-p_{H f}\right) \geq T_{H} .
\end{aligned}
$$

Intuitively, the left-hand sides of both $\left(\overline{I C_{L}}\right)$ and $\left(\overline{I C_{H}}\right)$ are the additional benefits by implementing treatment $T_{H}$ and the right-hand sides the additional costs. The expert will indeed implement treatment $T_{H}$ if the additional benefits exceed the additional treatment costs. Due to (13), the constraint $\left(\overline{I C_{L}}\right)$ implies the constraint $\left(\overline{I C_{H}}\right)$. Therefore, the contractual payments are incentive compatible as long as the constraint $\left(I C_{L}\right)$ is satisfied. The logic is that upon observing a signal $s_{L}$ the expert believes that the consumer's problem is more likely minor. Then the additional benefits from honestly implementing treatment $T_{H}$ are smaller than that upon observing a signal $s_{H}$. Therefore, if the expert prefers to implement treatment $T_{H}$ after a signal $s_{L}$, he will certainly do so also after a signal $s_{H}$.

For the expert to accept the contract, he has to earn a non-negative profit under the contract, so that $\Pi\left(T_{H}\right) \geq 0$, which is equivalent to

$$
\rho p_{H s}+(1-\rho) p_{H f}-T_{H} \geq 0
$$

In addition, the expert is protected by limited liability that all payments must be nonnegative:

$$
p_{H s} \geq 0, \quad p_{H f} \geq 0
$$


To optimally implement $\mathbf{H}$, the consumer maximizes his expected surplus

$$
S_{H}=\rho L-\rho p_{H s}-(1-\rho) p_{H f}
$$

subject to the constraints $\left(\overline{I C_{L}}\right),(\underline{I R})$, and $(\underline{L L})$. In the Appendix we prove the following result:

Proposition 3. The optimal contract to implement treatment strategy $\boldsymbol{H}$ has the following properties:

(a) The optimal payments are

$$
p_{H f}=0, \quad p_{H s}=\frac{T_{H}}{\rho\left(1-\mu_{L}\right)}
$$

(b) The expert's profits and the consumer's surplus are

$$
\Pi\left(T_{H}\right)=\frac{\mu_{L} T_{H}}{1-\mu_{L}}, \quad S_{H}=\rho L-\frac{T_{H}}{1-\mu_{L}} .
$$

Under the optimal contract, only the limited liability constraint in state of failure is binding: the expert is punished by zero payment when the treatment fails. Meanwhile, to induce the expert to indeed implement treatment $T_{H}$, the contract rewards him with a net profit when the treatment succeeds. As a result, the expert receives a strictly positive limited liability rent $\Pi\left(T_{H}\right)>0$. This is a standard result in principal-agent models with moral hazard and limited liability (see e.g. Sappington (1983)). An interesting insight in our model, however, is that the expert's rents increase with the accuracy of the signal because

$$
\frac{\partial \Pi\left(T_{H}\right)}{\partial \sigma}=\frac{T_{H}}{\left(1-\mu_{L}\right)^{2}} \cdot \frac{\partial \mu_{L}}{\partial \sigma}>0
$$

Intuitively, when the signal becomes more precise, upon observing a signal $s_{L}$ the expert is more inclined to implement treatment $T_{L}$ rather than treatment $T_{H}$ as required. In turn, the optimal contract has to provide a stronger incentive for the expert to choose treatment $T_{H}$, i.e. a larger reward for success. As this raises the rents of the expert, a more precise diagnosis is detrimental for the consumer for the implementation of $\mathbf{H}$.

Finally, we turn to the third case $\mathbf{D}=\mathbf{S}$ in which the consumer wants to elicit truthful reporting and implementing a treatment $T_{i}$ in accordance with signal $s_{i}$. Again, by the Revelation Principle of Myerson (1979, 1982), we can restrict ourselves to incentive compatible contracts under which the expert will not only report truthfully but also implement the treatment based on the signal. Assume that the consumer offers a contract $\left\{\left(p_{L s}, p_{L f}\right),\left(p_{H s}, p_{H f}\right)\right\}$. When the expert reports a signal $\hat{s}_{i}$ with $i \in\{H, L\}$, he receives the payment $p_{i s}$ if the treatment succeeds, and $p_{i f}$ otherwise.

After observing the signal, the expert's strategy consists of reporting a signal $\hat{s}_{i} \in\left\{\hat{s}_{L}, \hat{s}_{H}\right\}$ and choosing a treatment $T_{j} \in\left\{T_{L}, T_{H}\right\}$, denoted as a combination $\left(\hat{s}_{i}, T_{j}\right)$. Therefore, after each signal, there are four options available for the expert. We denote by $\Pi\left(\hat{s}_{i}, T_{j} \mid s_{i}\right)$ the expert's expected profits from choosing a combination $\left(\hat{s}_{i}, T_{j}\right)$ upon observing a signal $s_{i}$. 
After signal $s_{L}$ the expert will indeed report signal $\hat{s}_{L}$ and choose treatment $T_{L}$, if this strategy is at least as profitable as any other. In other words, the contractual payments must satisfy the following incentive compatibility constraints after signal $s_{L}$,

$$
\Pi\left(\hat{s}_{L}, T_{L} \mid s_{L}\right) \geq \Pi\left(\hat{s}_{i}, T_{j} \mid s_{L}\right), \quad \text { for } i, j \in\{L, H\}
$$

which are equivalent to

$$
\begin{array}{ll}
\mu_{L} \rho p_{L s}+\left(1-\mu_{L} \rho\right) p_{L f}-T_{L} \geq \rho p_{L s}+(1-\rho) p_{L f}-T_{H}, & \left(I C_{L 1}\right) \\
\mu_{L} \rho p_{L s}+\left(1-\mu_{L} \rho\right) p_{L f}-T_{L} \geq \mu_{L} \rho p_{H s}+\left(1-\mu_{L} \rho\right) p_{H f}-T_{L}, & \left(I C_{L 2}\right) \\
\mu_{L} \rho p_{L s}+\left(1-\mu_{L} \rho\right) p_{L f}-T_{L} \geq \rho p_{H s}+(1-\rho) p_{H f}-T_{H} . & \left(I C_{L 3}\right)
\end{array}
$$

Note that the constraint $\left(\overline{I C_{L 2}}\right)$ ensures that the expert does not report signal $\hat{s}_{H}$ in combination with treatment $T_{L}$; the constraints $\left(\overline{I C_{L 1}}\right)$ and $\left(\overline{I C_{L 3}}\right)$ guarantee that the expert does not choose treatment $T_{H}$ after he reported a signal $\hat{s}_{L}$ or $\hat{s}_{H}$.

Similarly, upon observing the signal $s_{H}$ the expert will truthfully report $\hat{s}_{H}$ and choose treatment $T_{H}$ if the contractual payments satisfy the incentive compatibility constraints after signal $s_{H}$ :

$$
\Pi\left(\hat{s}_{H}, T_{H} \mid s_{H}\right) \geq \Pi\left(\hat{s}_{i}, T_{j} \mid s_{H}\right), \quad \text { for } i, j \in\{H, L\} .
$$

These constraints are equivalent to:

$$
\begin{array}{ll}
\rho p_{H s}+(1-\rho) p_{H f}-T_{H} \geq \mu_{H} \rho p_{L s}+\left(1-\mu_{H} \rho\right) p_{L f}-T_{L}, & \left(I C_{H 1}\right) \\
\rho p_{H s}+(1-\rho) p_{H f}-T_{H} \geq \rho p_{L s}+(1-\rho) p_{L f}-T_{H}, & \left(I C_{H 2}\right) \\
\rho p_{H s}+(1-\rho) p_{H f}-T_{H} \geq \mu_{H} \rho p_{H s}+\left(1-\mu_{H} \rho\right) p_{H f}-T_{L} . & \left(I C_{H 3}\right)
\end{array}
$$

Note that the constraint $\left(\overline{I C_{H 2}}\right)$ induces the expert not to report $\hat{s}_{L}$; the constraints $\left(I C_{H 1}\right)$ and $\left(I C_{H 3}\right)$ guarantee that the expert cannot gain by selecting treatment $T_{L}$ and reporting either $\hat{s}_{L}$ or $\hat{s}_{H}$.

For the expert to accept the contract, he has to earn a non-negative expected profit under the contract. We denote by $\lambda$ the ex ante probability that the expert observes a signal $s_{L}$ :

$$
\lambda:=q \sigma+(1-q)(1-\sigma) .
$$

Then the expert's ex ante participation constraint requires that

$$
\Pi\left(T_{S}\right)=\lambda \Pi\left(\hat{s}_{L}, T_{L} \mid s_{L}\right)+(1-\lambda) \Pi\left(\hat{s}_{H}, T_{H} \mid s_{H}\right) \geq 0,
$$

because with probability $\lambda$ he observes signal $s_{L}$ and obtains the profit $\Pi\left(\hat{s}_{L}, T_{L} \mid s_{L}\right)$ by a combination $\left(\hat{s}_{L}, T_{L}\right)$, and with probability $1-\lambda$ his profit is $\Pi\left(\hat{s}_{H}, T_{H} \mid s_{H}\right)$ after signal $s_{H}$. In addition, all payments must be nonnegative:

$$
p_{L s} \geq 0, \quad p_{L f} \geq 0, \quad p_{H s} \geq 0, \quad p_{H f} \geq 0,
$$

because the expert is protected by limited liability. 
To optimally implement strategy $\mathbf{S}$, the payments $\left\{\left(p_{L s}, p_{L f}\right),\left(p_{H s}, p_{H f}\right)\right\}$ have to maximize the consumer's expected surplus

$$
\begin{aligned}
S_{S}= & \lambda\left[\mu_{L} \rho L-\mu_{L} \rho p_{L s}-\left(1-\mu_{L} \rho\right) p_{L f}\right] \\
& +(1-\lambda)\left[\rho L-\rho p_{H s}-(1-\rho) p_{H f}\right]
\end{aligned}
$$

subject to the incentive constraints $\left(\underline{I C_{L 1}}\right)-\left(\sqrt[I C_{L 3}]{ }\right),\left(\left[C_{H 1}\right)-\left(I C_{H 3}\right)\right.$, the participation constraint $(I R)$, and the limited liability constraint $(L L)$. In the Appendix we derive the following result on the solution of this problem:

Proposition 4. The optimal contract to implement treatment strategy $S$ has the following properties:

(a) The optimal payments are

$$
p_{L f}=p_{H f}=0, \quad p_{L s}=p_{H s}=\frac{T_{H}}{\rho\left(1-\mu_{H}\right)} .
$$

(b) The expert's profits and the consumer's surplus are

$$
\Pi\left(T_{S}\right)=\frac{q T_{H}}{1-\mu_{H}}, \quad S_{S}=\left[\lambda \mu_{L}+(1-\lambda)\right]\left[\rho L-\frac{T_{H}}{1-\mu_{H}}\right] .
$$

In Proposition 4, the optimal contractual payments depend not on the expert's report but only on the treatment outcome. When the treatment outcomes are the same, the expert receives the same payments regardless of his report and treatment. Thus, in some sense overcharging is unavoidable: in the event of success the expert receives a payment which is higher than the cost of the high-cost treatment $T_{H}$ even when he performs only the low-cost treatment $T_{L}$. The reason is the adverse selection problem related to the expert's report. With payments that depend on his report, the expert would always report the signal that gives him a larger profit.

Similar to Proposition 3, under the optimal contract the limited liability constraint in state of failure is binding: the expert receives zero payment when the treatment fails. When the treatment succeeds, however, the expert is rewarded by a net profit. This motivates him to select treatment $T_{H}$ after signal $s_{H}$. As a result, the expert also earns a positive expected rent $\Pi\left(T_{S}\right)>0$. In contrast with $(25)$, however, here it decreases with the accuracy of the signal as

$$
\frac{\partial \Pi\left(T_{S}\right)}{\partial \sigma}=\frac{q T_{H}}{\left(1-\mu_{H}\right)^{2}} \cdot \frac{\partial \mu_{H}}{\partial \sigma}<0 .
$$

Intuitively, given that the expert reports truthfully, the contractual payments must induce the expert to implement treatment $T_{H}$ only after signal $s_{H}$. When the signal becomes more precise, signal $s_{H}$ indicates problem $\theta_{H}$ with larger confidence, and so deviating to treatment $T_{L}$ becomes less attractive for the expert. Thus, motivating the expert to select $T_{H}$ after $s_{H}$ becomes easier, and this leads to a smaller rent for the expert.

This means that the consumer enjoys not only the efficiency gains from better information but also the reduction of the expert's rent. Indeed, we have

$$
\frac{\partial S_{S}}{\partial \sigma}=(1-q)\left(\rho L-\frac{T_{H}}{1-\mu_{H}}\right)-\frac{(1-\lambda) T_{H}}{\left(1-\mu_{H}\right)^{2}} \cdot \frac{\partial \mu_{H}}{\partial \sigma}-\frac{q T_{H}}{\left(1-\mu_{H}\right)^{2}} \cdot \frac{\partial \mu_{H}}{\partial \sigma}>0,
$$


because, as long as $S_{S}>0$, all the three terms are strictly positive: the first two terms are the benefits of a more precise signal from reducing undertreatment and overtreatment; the third one is the benefit from alleviating the moral hazard problem. As a result, differently from the situation in Proposition 3, here the consumer is better off if the expert is better informed.

\section{Optimal Contracts}

Propositions 2, 3, and 4 characterize the optimal contracts to implement a given treatment strategy $\mathbf{D} \in\{\mathbf{L}, \mathbf{H}, \mathbf{S}\}$. In this section, we derive the overall optimal contract by comparing the consumer's expected surplus from these treatment strategies.

We compare first the desirability of implementing treatments $\mathbf{L}$ and $\mathbf{H}$, under which the consumer does not seek the expert's advice. Let $S_{L H}(q)$ be the difference of the consumer's surplus from implementing treatment $T_{L}$ and treatment $T_{H}$. From Propositions 2 and 3 we obtain

$$
S_{L H}(q) \equiv S_{L}-S_{H}=\frac{T_{H}}{1-\mu_{L}}-(1-q) \rho L .
$$

Implementing treatment $T_{L}$ is thus at least as good as treatment $T_{H}$ if $S_{L H}(q) \geq 0$. Note that $S_{L H}(q)$ is increasing in $q$ because

$$
\frac{\partial S_{L H}(q)}{\partial q}=\frac{(1-q)^{2}(\sigma-1) \rho L-\sigma T_{H}}{(1-q)^{2}(\sigma-1)}>0 .
$$

Intuitively, the risk of overtreatment with treatment $T_{H}$ increases in $q$, while the risk of undertreatment with treatment $T_{L}$ decreases in $q$. Therefore, implementing treatment $T_{L}$ becomes more attractive as $q$ increases. Since $S_{L H}(0)=T_{H}-\rho L<0$ and $S_{L H}(q)>0$ for $q$ close enough to unity, there exists an unique $\hat{q} \in(0,1)$ such that

$$
S_{L H}(\hat{q})=0 .
$$

This means that implementing treatment $\mathbf{L}$ is better than $\mathbf{H}$ for $q>\hat{q}$; for $q<\hat{q}$ implementing treatment $\mathbf{H}$ is better. This result is similar to the role of $\bar{q}$ in $(8)$ in the first-best. But, it is easily verified that ${ }^{18}$

$$
\hat{q}<\bar{q} .
$$

Therefore, in comparison with the first-best, implementing treatment $T_{H}$ is desirable for a smaller range of priors. The reason is that by Proposition 3 treatment $T_{H}$ must entail a positive rent for the expert. We summarize the result of this comparison in the following proposition.

Proposition 5. When treatment choice is nonobservable, implementing treatment strategy $\boldsymbol{L}$ is at least as good as $\boldsymbol{H}$ if and only if $q \geq \hat{q}$; otherwise implementing strategy $\boldsymbol{H}$ is better. Compared with the first-best, the consumer chooses less often to implement strategy $\boldsymbol{H}$ because $\hat{q}<\bar{q}$.

\footnotetext{
${ }^{18}$ This directly follows from $\partial S_{L H}(q) / \partial q>0$ and $S_{L H}(\bar{q})=\mu_{L} T_{H} /\left(1-\mu_{L}\right)>S_{L H}(\hat{q})=0$.
} 


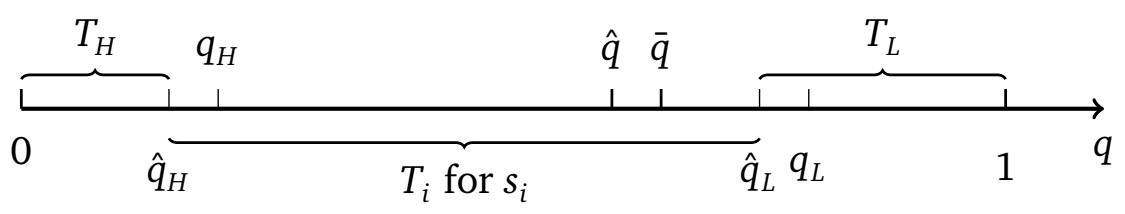

Figure 2: Optimal Treatments

We proceed by comparing treatment strategies $\mathbf{L}$ and $\mathbf{S}$. Define $S_{L S}(q)$ as the difference of the consumer's surplus from these two strategies:

$$
S_{L S}(q) \equiv S_{L}-S_{S}=\left(\lambda \mu_{L}+1-\lambda\right) \frac{T_{H}}{1-\mu_{H}}-(1-\lambda)\left(1-\mu_{H}\right) \rho L,
$$

where the first term represents the additional cost of treatment strategy $\mathbf{S}$, as it involves treatment $T_{H}$ with positive probability, and the second its additional benefits. Similarly, to compare treatment strategies $\mathbf{H}$ and $\mathbf{S}$, let $S_{H S}(q)$ denote the difference of the consumer's surplus from strategies $\mathbf{H}$ and $\mathbf{S}$ :

$$
S_{H S}(q) \equiv S_{H}-S_{S}=\lambda\left(1-\mu_{L}\right) \rho L-\left[\frac{T_{H}}{1-\mu_{L}}-\left(\lambda \mu_{L}+1-\lambda\right) \frac{T_{H}}{1-\mu_{H}}\right],
$$

where the first term is the additional benefit of treatment strategy $\mathbf{H}$ and the second its additional cost.

Implementing treatment strategy $\mathbf{S}$ is at least as good as implementing strategy $\mathbf{L}$ or $\mathbf{H}$ if and only if

$$
S_{L S}(q) \leq 0 \quad \text { and } \quad S_{H S}(q) \leq 0 .
$$

Analogously to the critical values $q_{L}$ and $q_{H}$ in (9) and (10) for the analysis of the first-best, we denote by $\hat{q}_{L}$ and $\hat{q}_{H}$ the values of the prior where ${ }^{19}$

$$
S_{L S}\left(\hat{q}_{L}\right)=0, \quad S_{H S}\left(\hat{q}_{H}\right)=0 .
$$

The following proposition, which we prove in the Appendix, characterizes the overall optimal contract for the consumer.

Proposition 6. When the signal is the expert's private information and treatment choice is unobservable, the optimal contract has the following properties:

(a) For $q>\hat{q}_{L}$, treatment strategy $L$ is optimal with the payments in Proposition 2 . Further, $\hat{q}_{L}<q_{L}$.

(b) For $q<\hat{q}_{H}$, treatment strategy $\boldsymbol{H}$ is optimal with the payments in Proposition 3 Further, $\hat{q}_{H}<q_{H}$.

(c) For $\hat{q}_{H} \leq q \leq \hat{q}_{L}$, treatment strategy $S$ is optimal with the payments in Proposition 4.

Figure 2 illustrates the results in Proposition 6 and compares them with the first-best in Proposition 1 . For values of the prior $q$ in the interval $\left[\hat{q}_{H}, \hat{q}_{L}\right]$, the optimal contract uses the

\footnotetext{
${ }^{19}$ Note that, as we show below, $\hat{q}_{H}>0$ only if $\sigma$ is not too close to unity
} 
expert's information and implements treatment $T_{i}$ after signal $s_{i}$. Outside this interval, the treatment does not depend on the expert's information: treatment $T_{H}$ is optimal if $q<\hat{q}_{H}$, and treatment $T_{L}$ is optimal if $q>\hat{q}_{L}$.

Since $\hat{q}_{L}<q_{L}$, there is a distortion relative to the first-best in the nonempty interval $\left[\hat{q}_{L}, q_{L}\right]$. Within this interval the consumer chooses to implement $T_{L}$, whereas in the first-best the treatment choice would depend on the expert's signal. While in the first-best treatment $T_{H}$ would be selected after $s_{H}$, under the optimal contract $T_{L}$ is chosen instead. In this sense, there is a positive probability of undertreatment when $q \in\left(\hat{q}_{L}, q_{L}\right]$. The reason is, of course, that treatment strategy $\mathbf{S}$ has to leave a rent to the expert, which makes this strategy less attractive than $\mathbf{L}$.

Similarly, undertreatment occurs also for values of $q$ in the nonempty interval $\left[\hat{q}_{H}, q_{H}\right]$. Here the optimal contract implements $T_{L}$ after the expert observes $s_{L}$. In the first-best, however, treatment $T_{H}$ would always be chosen. The intuition is that under both treatment strategies $\mathbf{S}$ and $\mathbf{H}$ the expert receives a rent. But, the expert's rent is higher under $\mathbf{H}$ than under $\mathbf{S}$. This induces the consumer to rely on the expert's information rather than to mandate treatment $T_{H}$.

We conclude this section by studying the optimal contract in the limiting case $\sigma \rightarrow 1$, in which the signal becomes perfectly precise. Note that

$$
\lim _{\sigma \rightarrow 1} \lambda=1, \quad \lim _{\sigma \rightarrow 1} \mu_{L}=1, \quad \lim _{\sigma \rightarrow 1} \mu_{H}=0 .
$$

Therefore,

$$
\lim _{\sigma \rightarrow 1} S_{L S}=T_{H}-(1-q) \rho L, \quad \lim _{\sigma \rightarrow 1} S_{H S}=-\infty .
$$

By the first equality in (41) and (39) we obtain that $\lim _{\sigma \rightarrow 1} \hat{q}_{L}=1-T_{H} / \rho L$. The second equality in (41) implies that for $\sigma$ close enough to unity treatment strategy $\mathbf{H}$ is always inferior to $\mathbf{S}$. This is so because $\mathbf{H}$ would require the expert to choose treatment $T_{H}$ also after observing signal $s_{L}$. As the signal becomes perfectly precise, however, the expert knows almost for sure that the consumer's problem is $\theta_{L}$ after signal $s_{L}$. Therefore, he knows that the low-cost treatment $T_{L}$ is almost certainly sufficient. Consequently, implementing $T_{H}$ independently of the expert's information becomes not only almost impossible but also undesirable for the consumer.

The following proposition summarizes our observations for the overall optimal contract for the limiting case $\sigma \rightarrow 1$.

Proposition 7. In the limit $\sigma \rightarrow 1$ treatment strategy $S$ is optimal if $q<1-T_{H} / \rho L$, otherwise strategy $L$ is optimal.

As illustrated in Figure 3, also in the limit $\sigma \rightarrow 1$ the overall optimal contract involves undertreatment. This happens for $q \in\left(1-T_{H} / \rho L, 1\right)$, where the first-best is strategy $\mathbf{S}$ but the optimal contract implements $\mathbf{L}$.

\section{Separation of Diagnosis and Treatment}

In this section, we explore whether separating diagnosis and treatment can reduce the inefficiencies of the optimal contract derived in the previous section. Indeed, separating 


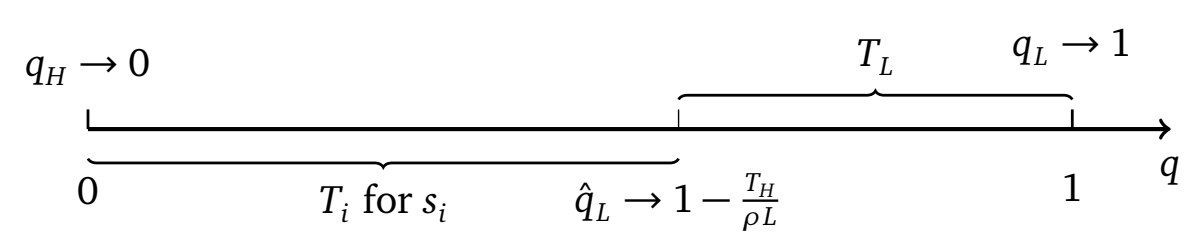

Figure 3: Optimal Treatments for $\sigma \rightarrow 1$

diagnosis and treatment is feasible in some situations. For example, in the medical services prescribing and selling drugs can be performed by different agents. ${ }^{20}$ The idea that this may reduce incentive problems in the provision of credence goods has been discussed already by Darby and Karni (1973): the expert performing the diagnosis may have less incentives to give wrong advice if he does not profit from performing the treatment.

We assume in this section that diagnosis and treatment are two independent procedures and that the consumer can contract with two different experts, $d$ and $t$, for diagnosis and treatment. Expert $d$ is an expert for diagnosis and can acquire the signal about the consumer's problem at zero cost; expert $t$ is an expert for treatment and can provide treatment $T_{i}$ by incurring the cost $T_{i} \in\left\{T_{H}, T_{L}\right\}$. After observing the signal, expert $d$ makes a report or prescription, which is observable to both the consumer and the expert $t$. Otherwise, the sequence of the events and the assumptions on observability are the same as in Section 2 .

Treatment Implementation As in our previous analysis, there are three treatment strategies $\mathbf{D} \in\{\mathbf{L}, \mathbf{H}, \mathbf{S}\}$. Since diagnosing is costless for expert $d$, the consumer's payment to him is optimally set equal to zero. Under this payment expert $d$ will truthfully report the signal, because he cannot benefit from misrepresenting. Consequently, not only the treatment expert $t$ but also the consumer can now observe the diagnosis signal at zero cost. Effectively, the situation is the same as if the consumer could perform the diagnosis by himself. As a result, the consumer and the expert $t$ hold the same posterior belief as in (12) after the reported signal. Since there is no longer an adverse selection problem of truthful reporting, we need to consider contracting only between the consumer and the treatment expert $t$.

In the same way as in Section 4, we begin by deriving the optimal implementation of a given strategy $\mathbf{D} \in\{\mathbf{L}, \mathbf{H}, \mathbf{S}\}$. First, consider strategy L: Since this strategy does not make use of the diagnosis, Proposition 2 also applies under separation and the consumer appropriates all economic rents.

Second, we consider treatment strategy $\mathbf{H}$. Even though under this strategy treatment $T_{H}$ is implemented independently of the information revealed by the diagnosis, it turns out that the consumer now can improve upon the optimal contract in Proposition 3 by offering a contract contingent both on the treatment outcome and on the signal. Assume that the consumer offers a contract $\left\{\left(p_{L s}, p_{L f}\right),\left(p_{H s}, p_{H f}\right)\right\}$. When the signal is $s_{i}$, expert $t$ receives the payment $p_{i s}$ if the treatment succeeds and $p_{i f}$ otherwise, with $i \in\{H, L\}$. Then, upon observing signal $s_{L}$ or $s_{H}$, by choosing treatment $T_{H}$ as required the treatment expert $t$ obtains

\footnotetext{
${ }^{20}$ See Dulleck and Kerschbamer $(2006)$ for more examples where separation is feasible or not.
} 
the expected profit

$$
\begin{aligned}
& \Pi\left(T_{H} \mid s_{L}\right)=\rho p_{L s}+(1-\rho) p_{L f}-T_{H}, \\
& \Pi\left(T_{H} \mid s_{H}\right)=\rho p_{H s}+(1-\rho) p_{H f}-T_{H},
\end{aligned}
$$

as treatment $T_{H}$ always succeeds with probability $\rho$. If however expert $t$ chose $T_{L}$ instead, then based on the posterior belief in (12) his expected profit would be

$$
\begin{aligned}
& \Pi\left(T_{L} \mid s_{L}\right)=\mu_{L} \rho p_{L s}+\left(1-\mu_{L} \rho\right) p_{L f}-T_{L}, \\
& \Pi\left(T_{L} \mid s_{H}\right)=\mu_{H} \rho p_{H s}+\left(1-\mu_{H} \rho\right) p_{H f}-T_{L},
\end{aligned}
$$

because treatment $T_{L}$ solves only problem $\theta_{L}$ with probability $\rho$.

Therefore, the expert will indeed choose treatment $T_{H}$ after signal $s_{L}$ and signal $s_{H}$ if and only if

$$
\Pi\left(T_{H} \mid s_{L}\right) \geq \Pi\left(T_{L} \mid s_{L}\right), \quad \Pi\left(T_{H} \mid s_{H}\right) \geq \Pi\left(T_{L} \mid s_{H}\right) .
$$

Equivalently, the contractual payments must satisfy the following two incentive compatibility constraints:

$$
\begin{aligned}
& \rho\left(1-\mu_{L}\right)\left(p_{L s}-p_{L f}\right) \geq T_{H}, \\
& \rho\left(1-\mu_{H}\right)\left(p_{H s}-p_{H f}\right) \geq T_{H} .
\end{aligned}
$$

In addition, for the expert to accept the contract, he has to earn a non-negative expected profit under the contract. Since his expected profit is

$$
\Pi^{s}\left(T_{H}\right) \equiv \lambda \Pi\left(T_{H} \mid s_{L}\right)+(1-\lambda) \Pi\left(T_{H} \mid s_{H}\right),
$$

the contract must satisfy the ex ante participation constraint:

$$
\lambda\left[\rho p_{L s}+(1-\rho) p_{L f}\right]+(1-\lambda)\left[\rho p_{H s}+(1-\rho) p_{H f}\right]-T_{H} \geq 0
$$

Finally, we have the constraints

$$
p_{i s} \geq 0, \quad p_{i f} \geq 0 \quad \text { for } i \in\{L, H\},
$$

because the expert is protected by limited liability.

To optimally implement treatment strategy $\mathbf{H}$, the consumer maximizes his expected surplus

$$
S_{H}^{s}=\rho L-\lambda\left[\rho p_{L s}+(1-\rho) p_{L f}\right]-(1-\lambda)\left[\rho p_{H s}+(1-\rho) p_{H f}\right]
$$

subject to the constraints $\left(I C_{L}\right),\left(\left[C_{H}\right),(I R)\right.$, and $(L L)$. In the Appendix we prove the following result:

Proposition 8. When diagnosis and treatment are separated, the optimal contract to implement treatment strategy $\boldsymbol{H}$ has the following properties:

(a) The optimal payments are

$$
p_{L f}=p_{H f}=0, \quad p_{L s}=\frac{T_{H}}{\rho\left(1-\mu_{L}\right)}, \quad p_{H s}=\frac{T_{H}}{\rho\left(1-\mu_{H}\right)} .
$$


(b) The profits of the expert $t$ and the consumer's surplus are

$$
\Pi^{s}\left(T_{H}\right)=\left[\frac{\lambda \mu_{L}}{1-\mu_{L}}+\frac{(1-\lambda) \mu_{H}}{1-\mu_{H}}\right] T_{H}, \quad S_{H}^{s}=\rho L-\left[\frac{\lambda}{1-\mu_{L}}+\frac{(1-\lambda)}{1-\mu_{H}}\right] T_{H} .
$$

When diagnosis and treatment are separated, the consumer can retain more economic rents by the truthful signal obtained from expert $d$. Indeed the consumer surplus $S_{H}^{s}$ in Proposition 8 is higher than the consumer surplus $S_{H}$ in Proposition 3 because $\mu_{L}>\mu_{H}$. The intuition is that the consumer's knowledge of the signal softens the incentive constraint after signal $s_{H}$. Therefore, the optimal payment $p_{H s}$ is lower than $p_{L s}$. While the $t$-expert's rent is decreased relative the situation without separation, it is still positive. Also it increases with the accuracy of the signal since

$$
\frac{\partial \Pi^{s}\left(T_{H}\right)}{\partial \sigma}=\frac{q^{2}(2 \sigma-1)}{(1-q) \sigma^{2}(1-\sigma)^{2}} T_{H}>0
$$

similarly to the case without separation in (25).

Third, to implement treatment strategy $\mathbf{S}$ the consumer offers the payments $\left\{\left(p_{L s}, p_{L f}\right),\left(p_{H s}, p_{H f}\right)\right\}$, with the same interpretation as above. These payments have to satisfy the incentive constraints that expert $t$ will indeed choose treatment $T_{i}$ after signal $s_{i}$

$$
\Pi\left(T_{L} \mid s_{L}\right) \geq \Pi\left(T_{H} \mid s_{L}\right), \quad \Pi\left(T_{H} \mid s_{H}\right) \geq \Pi\left(T_{L} \mid s_{H}\right),
$$

which are equivalent to

$$
\begin{aligned}
& \mu_{L} \rho p_{L s}+\left(1-\mu_{L} \rho\right) p_{L f} \geq \rho p_{L s}+(1-\rho) p_{L f}-T_{H} \\
& \rho p_{H s}+(1-\rho) p_{H f}-T_{H} \geq \mu_{H} \rho p_{H s}+\left(1-\mu_{H} \rho\right) p_{H f}
\end{aligned}
$$

In addition, the contract must also satisfy the same participation constraint as in $(\overline{I R})$ and the same limited liability constraints as in $(L L)$.

Note that the formula for the consumer's surplus $S_{S}^{s}$ from implementing $\mathbf{S}$ under separation is the same as for $S_{S}$ in (27). The following result, which is proven in the Apppendix, characterizes the solution of the consumer's problem to maximize $S_{S}^{s}$ subject to $\left(I C_{L}\right),\left(I C_{H}\right),(I R)$ and $(L L)$ :

Proposition 9. When diagnosis and treatment are separated, the optimal contact to implement treatment strategy $S$ has the following properties:

(a) The optimal payments are:

$$
p_{L s}=p_{L f}=p_{H f}=0, \quad p_{H s}=\frac{T_{H}}{\rho\left(1-\mu_{H}\right)} .
$$

(b) The profits of expert $t$ and the consumer's expected surplus are:

$$
\Pi^{s}\left(T_{S}\right)=(1-\lambda) \frac{\mu_{H} T_{H}}{1-\mu_{H}}, \quad S_{S}^{s}=\left(\lambda \mu_{L}+1-\lambda\right) \rho L-\frac{(1-\lambda) T_{H}}{1-\mu_{H}} .
$$


As in the previous proposition, under separation the consumer can ensure himself a higher surplus compared with Proposition 4 as $S_{S}^{s}>S_{S}$. This is so because separation allows the consumer to keep the rent for inducing truthful reporting. Still, there remains a positive rent for expert $t$ to motivate him implementing treatment $T_{H}$ after a signal $s_{H}$. His rent, however, decreases with the accuracy of the signal because

$$
\frac{\partial \Pi^{s}\left(T_{S}\right)}{\partial \sigma}=-\left[q+\frac{q^{2}(1-\sigma)^{2}}{(1-q) \sigma^{2}}\right] T_{H}<0 .
$$

The intuition is the same as for the inequality in $(30)$ in the case without separation.

Optimal Contracts We can now compare the consumer's surplus in Propositions 2, 8 and 9 to derive the optimal treatment strategy for the consumer when diagnosis and treatment are separately provided by two experts.

Let $S_{L S}^{s}(q)$ be the difference of the consumer's surplus from treatment strategies $\mathbf{L}$ and $\mathbf{S}$ under separation of diagnosis and treatment:

$$
S_{L S}^{s}(q) \equiv S_{L}-S_{S}^{s}=(1-\lambda) \frac{\mu_{H} T_{H}}{1-\mu_{H}}+(1-\lambda) T_{H}-(1-\lambda)\left(1-\mu_{H}\right) \rho L .
$$

Similarly, let $S_{H S}^{s}(q)$ be the difference of the consumer's surplus from $\mathbf{H}$ and $\mathbf{S}$ :

$$
S_{H S}^{s}(q) \equiv S_{H}^{s}-S_{S}^{s}=\lambda\left(1-\mu_{L}\right) \rho L-\frac{\lambda T_{H}}{1-\mu_{L}}
$$

We denote by $q_{L}^{s}$ and $q_{H}^{s}$ the critical values of the prior where

$$
S_{L S}^{s}\left(q_{L}^{s}\right)=0, \quad S_{H S}^{s}\left(q_{H}^{s}\right)=0
$$

In the following result, which we prove in the Appendix, these critical values play the same role as the critical values $q_{L}$ and $q_{H}$ in (9) and (10) for the first-best, and $\hat{q}_{L}$ and $\hat{q}_{H}$ in (39) for the optimal contract without separation. To state the result, we define the parameter

$$
\gamma \equiv(1+\sqrt{5}) / 2 \approx 1.61803
$$

which is known in mathematics as the "golden ratio" or the "golden mean".

Proposition 10. When diagnosis and treatment are separated, the optimal contract has the following properties:

(a) For $q>q_{L}^{s}$, treatment strategy $L$ is optimal with the payments in Proposition 2. Further, $q_{L}>q_{L}^{s}>\hat{q}_{L}$.

(b) For $q<q_{H}^{s}$, treatment strategy $\boldsymbol{H}$ is optimal with the payments in Proposition 8 Further, $q_{H}>q_{H}^{s}>\hat{q}_{H}$ if $\sigma \in(\gamma-1,1)$, and $q_{H}>\hat{q}_{H}>q_{H}^{s}$ if $\sigma \in(1 / 2, \gamma-1)$.

(c) For $q_{H}^{s} \leq q \leq q_{L}^{s}$, treatment strategy $S$ is optimal with the payments in Proposition 9 . 


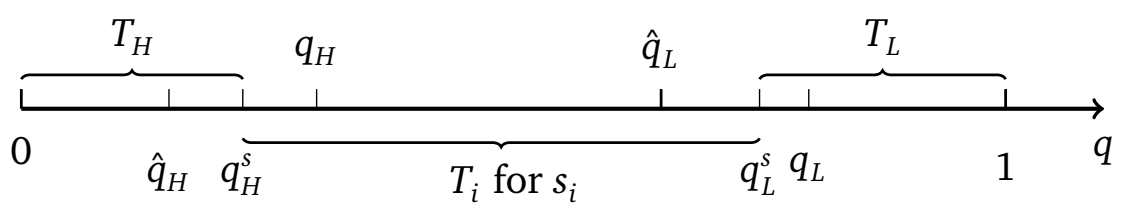

Case 1: $\sigma \in(\gamma-1,1)$

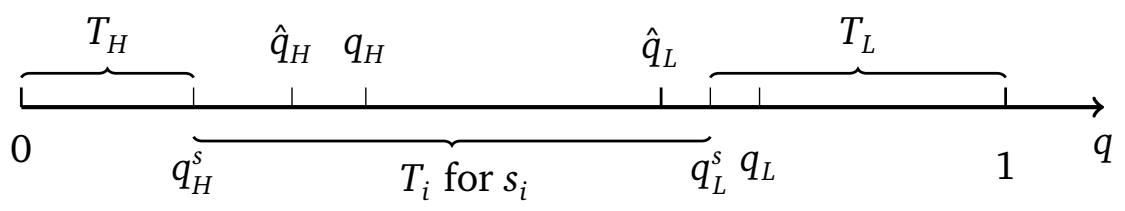

Case 2: $\sigma \in(1 / 2, \gamma-1)$

Figure 4: Optimal Treatments under Separation

Figure 4 illustrates Proposition 10. The qualitative features of the optimal contract are similar to the situation without separation in Proposition 6; the diagnosis signal plays a role for the selection of treatment only for intermediate values of the prior. If the prior is sufficiently informative, treatment $T_{H}$ is implemented for low values of the prior and $T_{L}$ for high values.

However, the two threshold values of the prior, $q_{L}^{s}$ and $q_{H}^{s}$, differ from $\hat{q}_{L}$ and $\hat{q}_{H}$ in Proposition 6. This implies that the likelihood of undertreatment is unambiguously reduced in the interval $q \in\left[\hat{q}_{L}, q_{L}^{s}\right]$, because only under separation the first-best strategy $\mathbf{S}$ is used. In case 1 in Figure 4, where the signal is relatively accurate, separation reduces the likelihood of undertreatment also in the interval $\left[\hat{q}_{H}, q_{H}^{s}\right]$, because only under separation the first-best strategy $\mathbf{H}$ is used. In contrast, if the signal is not very accurate as in case 2 , undertreatment is increased in the interval $q \in\left[q_{H}^{s}, \hat{q}_{H}\right]$, because separation makes strategy $\mathbf{S}$ optimal, whereas without separation the first-best strategy $\mathbf{H}$ is optimal. Thus, when the consumer's problem is more likely major for low values of $q$, the efficiency properties of separation are ambiguous as they depend on the precision of the diagnosis. Note, however, that from the consumer's perspective separation of diagnosis and treatment is always preferable. The reason is that, by Propositions 8 and 9, separation reduces the rents that the consumer has to leave to the expert under strategies $\mathbf{H}$ and $\mathbf{S}$.

Even though separation certainly reduces inefficient undertreatment for high values of $\sigma$, it cannot restore the first-best as $q_{L}^{s}<q_{L}$ and $q_{H}^{s}<q_{H}$. While separating diagnosis and treatment solves the adverse selection problem of the expert's private diagnosis information, the tradeoff between rent extraction and efficiency persists due to the moral hazard problem of unobservable treatment choice and limited liability. This explains the difference with Bester and Dahm (2017), who under the assumption of verifiable treatments show that separating diagnosis and treatment can always implement the first-best outcome. 


\section{Conclusion}

We have analyzed the optimal contract for a consumer to procure a credence good from an expert who obtains additional, but imprecise information about the consumer's need by a costless diagnosis. There are two incentive problems. First, the diagnosis result is the expert's private information. This creates an adverse selection problem of truthful reporting. Second, the expert's choice of treatment is not observable, which leads to a moral hazard problem of treatment selection. In addition, the expert is protected by limited liability so that penalty payments from the expert to the consumer are prohibited.

There are three feasible treatment strategies. Ex ante, before the expert diagnoses the problem, the consumer can offer a contract for implementing one of three treatment strategies. Whenever a high-cost treatment is part of a treatment strategy, implementing this strategy must entail a strictly positive rent for the expert. As a result, the strategy becomes less attractive for the consumer. We show that, compared with the first-best outcome, the optimal contract therefore leads to too much undertreatment. First, if the consumer's prior probability makes it highly likely that his problem is a minor one, he may refrain from using the expert's information by insisting on a low-cost treatment. For some values of the prior this is inefficient because in the first-best a high-cost treatment would be selected after the diagnosis indicates a major problem. Second, if the consumer's prior probability makes it highly likely that his problem is a major one, then in the first-best the high-cost treatment is efficient. Yet, under the optimal contract the consumer may choose to implement the low-cost treatment instead after the expert's imprecise diagnosis indicates a minor problem. In summary, relative to the first-best not only the implementation of treatment strategies may be inefficient but also the usage of the expert's information.

While in the main part of our analysis a single expert performs both diagnosis and treatment, we also consider the case where the two activities can be allocated to two separate experts. We show that this reduces the first type of inefficiency indicated above. For the second type of inefficiency indicated above, the result depends on the accuracy of the experts diagnosis: if it is highly accurate the inefficiency is reduced, otherwise it is increased. In any case, also with separation the optimal contract involves undertreatment for some parameter combinations. But from the consumer's perspective separation is always desirable because he has to leave less rents to the experts and so receives a higher expected surplus. 


\section{Appendix}

This appendix contains the proofs of Propositions 3, 46, and 8- 10, All other propositions are substantiated in the main text.

Proof of Proposition 3: Define $\Delta_{H} \equiv \rho\left(p_{H s}-p_{H f}\right)$. The consumer's program can then be rewritten as

$$
\max _{\left\{p_{H f}, \Delta_{H}\right\}} \rho L-p_{H f}-\Delta_{H}
$$

subject to the transformed constraints

$$
\begin{aligned}
& \left(1-\mu_{L}\right) \Delta_{H} \geq T_{H}, \\
& p_{H f}+\Delta_{H}-T_{H} \geq 0, \\
& p_{H f} \geq 0, \quad p_{H f}+\Delta_{H} \geq 0,
\end{aligned}
$$

Let us ignore the constraint $(I R)$ for the moment, which will be checked later. Note that the constraint $\left(I C_{L}\right)$ puts no further restriction on $p_{H f}$, then $p_{H f}=0$ follows immediately from the first inequality of constraint $(\overline{L L})$ as the consumer's surplus is strictly decreasing in $p_{H f}$.

Since the consumer's surplus is strictly decreasing in $\Delta_{H}$, it is optimal to set $\Delta_{H}=T_{H} /(1-$ $\left.\mu_{L}\right)$ to satisfy the constraint $\left(I C_{L}\right)$. Substituting $p_{H f}=0$ into $\Delta_{H}$, we get $p_{H s}=T_{H} / \rho\left(1-\mu_{L}\right)$. Therefore, the optimal payments are

$$
p_{H f}=0, \quad p_{H s}=\frac{T_{H}}{\rho\left(1-\mu_{L}\right)} .
$$

It remains to check this contract indeed satisfies the constraint $(I R)$. The expert's expected profits and the consumer's expected surplus under the contact are

$$
\Pi\left(T_{H}\right)=\frac{\mu_{L} T_{H}}{\left(1-\mu_{L}\right)}, \quad S_{H}=\rho L-\frac{T_{H}}{\left(1-\mu_{L}\right)},
$$

where $\Pi\left(T_{H}\right)>0$ by (13). This shows that the contract with the payments in (61) is optimal. Q.E.D.

Proof of Proposition 4: Define $\Delta_{f} \equiv p_{L f}-p_{H f}, \Delta_{L} \equiv \rho\left(p_{L s}-p_{L f}\right)$, and $\Delta_{H} \equiv \rho\left(p_{H s}-p_{H f}\right)$. Ignoring for the moment the constraint $(I R)$, the consumer's problem then is equivalent to

$$
\max _{\left\{p_{H f}, \Delta_{f}, \Delta_{L}, \Delta_{H}\right\}}-\left[p_{H f}+\lambda \Delta_{f}+\lambda \mu_{L} \Delta_{L}+(1-\lambda) \Delta_{H}\right]
$$

subject to the six transformed incentive constraints

$$
\begin{array}{ll}
\Delta_{L}\left(1-\mu_{L}\right) \leq T_{H}, & \left(I C_{L 1}\right) \\
\Delta_{f} \geq \mu_{L}\left(\Delta_{H}-\Delta_{L}\right), & \left(I C_{L 2}\right) \\
\Delta_{f} \geq \Delta_{H}-\mu_{L} \Delta_{L}-T_{H}, & \left(I C_{L 3}\right) \\
\Delta_{f} \leq \Delta_{H}-\mu_{H} \Delta_{L}-T_{H}, & \left(I C_{H 1}\right) \\
\Delta_{f} \leq \Delta_{H}-\Delta_{L}, & \left(I C_{H 2}\right) \\
\Delta_{H}\left(1-\mu_{H}\right) \geq T_{H}, & \left(I C_{H 3}\right)
\end{array}
$$


and the limited liability constraints

$$
p_{H f} \geq 0, \quad p_{H f}+\Delta_{f} \geq 0, \quad p_{H f}+\Delta_{H} \geq 0, \quad p_{H f}+\Delta_{f}+\Delta_{L} \geq 0 .
$$

Note that the constraints $\left(\underline{I C_{H 2}}\right)$ and $\left(\overline{I C_{L 3}}\right)$ imply that

$$
\Delta_{H}-\Delta_{L} \geq \Delta_{f} \geq \Delta_{H}-\mu_{L} \Delta_{L}-T_{H} \quad \Rightarrow \Delta_{L}\left(1-\mu_{L}\right) \leq T_{H},
$$

where the last inequality is exactly constraint $\left(I C_{L 1}\right)$. Thus, the constraint $\left(I C_{H 2}\right)$ and $\left(I C_{L 3}\right)$ imply the constraint $\left(I C_{L 1}\right)$. Moreover, the constraints $\left(I C_{H 1}\right)$ and $\left(I C_{L 2}\right)$ imply that

$$
\begin{aligned}
& \Delta_{H}-\mu_{H} \Delta_{L}-T_{H} \geq \Delta_{f} \geq \mu_{L}\left(\Delta_{H}-\Delta_{L}\right) \geq \mu_{H}\left(\Delta_{H}-\Delta_{L}\right) \\
& \Rightarrow \Delta_{H}\left(1-\mu_{H}\right) \geq T_{H}
\end{aligned}
$$

where the last inequality in the first line holds as $\mu_{L}>\mu_{H}$ by (13). Thus, the constraints $\left(I C_{H 1}\right)$ and $\left(I C_{L 2}\right)$ imply the constraint $\left(I C_{H 3}\right)$.

Since $p_{H f}$ is not constrained by the expert's incentive constraints and the consumer's surplus is decreasing in $p_{H f}$, it follows from the first requirement of the constraint $(L L)$ that $p_{H f}=0$. Therefore, the consumer's problem is reduced to choose the optimal triple $\left\{\Delta_{f}, \Delta_{L}, \Delta_{H}\right\}$ to maximize his expected surplus subject to the remaining four incentive constraints. In what follows, we first show that $\Delta_{f} \geq 0, \Delta_{L} \geq 0$, and $\Delta_{H} \geq 0$; then we solve for the optimal triple by employing the Lagrangian method.

First, combining the constraints $\left(\left[C_{L 2}\right)\right.$ and $\left(\left[C_{H 2}\right)\right.$, then

$$
\mu_{L}\left(\Delta_{H}-\Delta_{L}\right) \leq \Delta_{f} \leq \Delta_{H}-\Delta_{L} \Rightarrow \Delta_{f} \geq 0,
$$

as $\mu_{L} \in(q, 1]$ due to $(13)$. Second, by combining the constraint $\left(I C_{L 3}\right)$ and $\left(I C_{H 1}\right)$, we get

$$
\begin{aligned}
& \Delta_{H}-\mu_{L} \Delta_{L}-T_{H} \leq \Delta_{f} \leq \Delta_{H}-\mu_{H} \Delta_{L}-T_{H} \\
& \Rightarrow\left(\mu_{L}-\mu_{H}\right) \Delta_{L} \geq 0 \Rightarrow \Delta_{L} \geq 0,
\end{aligned}
$$

as $\mu_{L} \geq \mu_{H}$ by (13). Third, by the constraint $\left(\overline{I C_{H 3}}\right)$ we get $\Delta_{H} \geq T_{H} /\left(1-\mu_{H}\right)>0$.

The Lagrangian of the consumer's problem is

$$
\begin{aligned}
L= & -\left[\lambda \Delta_{f}+\lambda \mu_{L} \Delta_{L}+(1-\lambda) \Delta_{H}\right]+\phi_{1}\left(\Delta_{f}-\mu_{L} \Delta_{H}+\mu_{L} \Delta_{L}\right) \\
& +\phi_{2}\left(\Delta_{f}-\Delta_{H}+\mu_{L} \Delta_{L}+T_{H}\right)+\phi_{3}\left(\Delta_{H}-\mu_{H} \Delta_{L}-T_{H}-\Delta_{f}\right) \\
& +\phi_{4}\left(\Delta_{H}-\Delta_{L}-\Delta_{f}\right)
\end{aligned}
$$


By taking derivatives we obtain the Kuhn-Tucker Conditions:

$$
\begin{array}{lll}
\frac{\partial L}{\partial \Delta_{f}}=-\lambda+\phi_{1}+\phi_{2}-\phi_{3}-\phi_{4} \leq 0, & \Delta_{f} \geq 0 & \text { and } \Delta_{f} \frac{\partial L}{\partial \Delta_{f}}=0 ; \\
\frac{\partial L}{\partial \Delta_{L}}=-\lambda \mu_{L}+\phi_{1} \mu_{L}+\phi_{2} \mu_{L}-\phi_{3} \mu_{H}-\phi_{4} \leq 0, & \Delta_{L} \geq 0 & \text { and } \Delta_{L} \frac{\partial L}{\partial \Delta_{L}}=0 ; \\
\frac{\partial L}{\partial \Delta_{H}}=-(1-\lambda)-\phi_{1} \mu_{L}-\phi_{2}+\phi_{3}+\phi_{4} \leq 0, & \Delta_{H} \geq 0 & \text { and } \Delta_{H} \frac{\partial L}{\partial \Delta_{H}}=0 ; \\
\frac{\partial L}{\partial \phi_{1}}=\Delta_{f}-\mu_{L} \Delta_{H}+\mu_{L} \Delta_{L} \geq 0, & \phi_{1} \geq 0 & \text { and } \phi_{1} \frac{\partial L}{\partial \phi_{1}}=0 ; \\
\frac{\partial L}{\partial \phi_{2}}=\Delta_{f}-\Delta_{H}+\mu_{L} \Delta_{L}+T_{H} \geq 0, & \phi_{2} \geq 0 & \text { and } \phi_{2} \frac{\partial L}{\partial \phi_{2}}=0 ; \\
\frac{\partial L}{\partial \phi_{3}}=\Delta_{H}-\mu_{H} \Delta_{L}-T_{H}-\Delta_{f} \geq 0, & \phi_{3} \geq 0 & \text { and } \phi_{3} \frac{\partial L}{\partial \phi_{3}}=0 ; \\
\frac{\partial L}{\partial \phi_{4}}=\Delta_{H}-\Delta_{L}-\Delta_{f} \geq 0, & \phi_{4} \geq 0 & \text { and } \phi_{4} \frac{\partial L}{\partial \phi_{4}}=0 .
\end{array}
$$

We prove $\Delta_{f}=0$ by contradiction. Suppose that $\Delta_{f}>0$, then $\partial L / \partial \Delta_{f}=0$, which is equivalent to

$$
-\lambda+\phi_{1}+\phi_{2}-\phi_{3}-\phi_{4}=0
$$

From the constraint $\left(\left[C_{H 3}\right)\right.$, we know that $\Delta_{H} \geq T_{H} /\left(1-\mu_{H}\right)>0$. Since $\Delta_{H} \partial L / \partial \Delta_{H}=0$, then $\partial L / \partial \Delta_{H}=0$, which is equivalent to

$$
-(1-\lambda)-\phi_{1} \mu_{L}-\phi_{2}+\phi_{3}+\phi_{4}=0
$$

Combining and solving (69) and (70), we obtain that $\phi_{1}=1 /\left(1-\mu_{L}\right)>0$. As $\phi_{1} \partial L / \partial \phi_{1}=$ 0 , it must be that $\partial L / \partial \phi_{1}=0$, which is equivalent to

$$
\Delta_{f}-\mu_{L} \Delta_{H}+\mu_{L} \Delta_{L}=0
$$

By substituting (71) into $\partial L / \partial \phi_{4}$, we get

$$
\frac{\partial L}{\partial \phi_{4}}=\Delta_{H}-\Delta_{L}-\Delta_{f}=\left(\frac{1}{\mu_{L}}-1\right) \Delta_{f}>0
$$

Thus, $\phi_{4}=0$ because $\phi_{4} \partial L / \partial \phi_{4}=0$. Substituting $\phi_{4}=0$ into (69), we get

$$
\phi_{3}+\lambda=\phi_{1}+\phi_{2}
$$

Substituting (73) into $\partial L / \partial \Delta_{L} \leq 0$, then

$$
\frac{\partial L}{\partial \Delta_{L}}=\left(\mu_{L}-\mu_{H}\right) \phi_{3} \leq 0 \Rightarrow \phi_{3} \leq 0
$$

where the implication holds because $\mu_{L} \geq \mu_{H}$. In addition, as $\phi_{3} \geq 0$, it must hold that $\phi_{3}=0$. Replacing $\phi_{1}=1 /\left(1-\mu_{L}\right)$ and $\phi_{3}=0$ into (73), we obtain

$$
\phi_{2}=\lambda-\frac{1}{1-\mu_{L}}<0,
$$


where the inequality holds because $\lambda \in[0,1]$ and $\mu_{L} \in(q, 1]$. The inequality in (75) yields a contradiction to $\phi_{2} \geq 0$. Therefore, the supposition that $\Delta_{f}>0$ cannot hold and thus $\Delta_{f}=0$.

By substituting $\Delta_{f}=0$, the four incentive constraints are reduced to

$$
\begin{array}{ll}
\mu_{L}\left(\Delta_{H}-\Delta_{L}\right) \leq 0, & \left(I C_{L 2}\right) \\
\Delta_{H}-\mu_{L} \Delta_{L}-T_{H} \leq 0, & \left(I C_{L 3}\right) \\
\Delta_{H}-\mu_{H} \Delta_{L}-T_{H} \geq 0, & \left(I C_{H 1}\right) \\
\Delta_{H}-\Delta_{L} \geq 0 . & \left(I C_{H 2}\right)
\end{array}
$$

We can summarize the four constraints as

$$
\frac{T_{H}}{1-\mu_{H}} \leq \Delta_{H}=\Delta_{L} \leq \frac{T_{H}}{1-\mu_{L}} .
$$

Since the consumer's surplus is strictly decreasing in $\Delta_{L}$ and $\Delta_{H}$, it is maximized by setting $\Delta_{L}=\Delta_{H}=T_{H} /\left(1-\mu_{H}\right)$. The optimal triple $\left\{\Delta_{f}, \Delta_{L}, \Delta_{H}\right\}$ is therefore

$$
\Delta_{f}=0, \quad \Delta_{L}=\Delta_{H}=\frac{T_{H}}{1-\mu_{H}} .
$$

By combining the triple $\left\{\Delta_{f}, \Delta_{L}, \Delta_{H}\right\}$ and $p_{H f}=0$, we obtain the optimal contract

$$
p_{L f}=p_{H f}=0, \quad p_{L s}=p_{H s}=\frac{T_{H}}{\rho\left(1-\mu_{H}\right)} .
$$

It remains to check whether this contract satisfies the constraint $(I R)$. Under the contract, the expert's expected payoff equals to

$$
\Pi\left(T_{S}\right)=\left[\lambda \mu_{L}+(1-\lambda)\right] \frac{T_{H}}{1-\mu_{H}}-(1-\lambda) T_{H}=\frac{q T_{H}}{1-\mu_{H}}>0 .
$$

Therefore, the contract is optimal since it also satisfies the constraint $(I R)$.

Q.E.D.

Proof of Proposition 6: Note that $S_{L S}(q)$ is increasing in $q$, while $S_{H S}(q)$ is decreasing in $q$ for $q \in[0,1]$ because

$$
\begin{aligned}
& \frac{\partial S_{L S}(q)}{\partial q}=\frac{1-\mu_{H}+q \mu_{H}^{\prime}}{\left(1-\mu_{H}\right)^{2}} T_{H}+(1-\sigma) T_{H}+\sigma\left(\rho L-T_{H}\right)>0, \\
& \frac{\partial S_{H S}(q)}{\partial q}=(\sigma-1)\left(\rho L-T_{H}+\frac{1-\sigma}{\sigma} T_{H}\right)-\frac{(2 \sigma-1) T_{H}}{\sigma(1-\sigma)(1-q)^{2}}<0,
\end{aligned}
$$

by $\mu_{H}^{\prime} \equiv \partial \mu_{H} / \partial q>0$ and (13).

Since $S_{L S}(0)=\sigma\left(T_{H}-\rho L\right)<0$ and $S_{L S}(q) \rightarrow+\infty$ for $q \rightarrow 1$, by continuity there exists a unique $\hat{q}_{L} \in(0,1)$ such that

$$
S_{L S}\left(\hat{q}_{L}\right) \equiv 0 .
$$

In addition, as $\partial S_{L S}(q) / \partial q>0$ and $S_{L S}\left(q_{L}\right)=q_{L} T_{H} /\left(1-\mu_{H}\right)>S_{L S}\left(\hat{q}_{L}\right) \equiv 0$, it follows that

$$
q_{L}>\hat{q}_{L} .
$$


Similarly, since $S_{H S}(0)=(1-\sigma)\left(\rho L-T_{H}\right)>0$ and $S_{H S}(q) \rightarrow-\infty$ for $q \rightarrow 1$, by continuity there exists a unique $\hat{q}_{H} \in(0,1)$ such that

$$
S_{H S}\left(\hat{q}_{H}\right) \equiv 0 .
$$

Note also that

$$
q_{H}>\hat{q}_{H},
$$

because $\partial S_{H S}(q) / \partial q<0$ and $S_{H S}\left(q_{H}\right)=\left(\lambda \mu_{L}+1-\lambda\right)\left(\mu_{H}-\mu_{L}\right) T_{H} /\left(1-\mu_{H}\right)\left(1-\mu_{L}\right)<$ $S_{H S}\left(\hat{q}_{H}\right) \equiv 0$ since $(13)$.

It is easily verified, that

$$
\hat{q}_{H}<\hat{q}<\hat{q}_{L}
$$

Therefore, implementing the treatment strategy $\mathbf{S}$ is optimal if and only if

$$
\hat{q}_{H} \leq q \leq \hat{q}_{L}
$$

Outside this interval, implementing $\mathbf{H}$ is optimal for $q<\hat{q}_{H}$, while implementing $\mathbf{L}$ for $q>\hat{q}_{L}$. Q.E.D.

Proof of Proposition 8: Let us ignore the constraint $(I R)$ for the moment, which will be checked later. Then the consumer's problem can be divided into two symmetric cases, which are to minimize the expected payment to the expert $t$ after a signal $s_{L}$ and $s_{H}$, respectively. Define $\Delta_{i} \equiv \rho\left(p_{i s}-p_{i f}\right)$, with $i \in\{H, L\}$. Then the consumer's program can be rewritten as

$$
\min _{\left\{p_{i f}, \Delta_{i}\right\}} p_{i f}+\Delta_{i}
$$

subject to

$$
\begin{aligned}
& \left(1-\mu_{i}\right) \Delta_{i} \geq T_{i}, \\
& p_{i f} \geq 0, \quad p_{i f}+\Delta_{i} \geq 0 .
\end{aligned}
$$

Note that the constraint $\left(I C_{i}\right)$ puts no further restriction on $p_{i f}$. Thus $p_{i f}=0$ follows immediately from the first inequality of constraint $(L L)$. Furthermore, it is obvious that setting $\Delta_{i}=T_{H} /\left(1-\mu_{i}\right)$ is optimal as the payment is increasing in $\Delta_{i}$. By plugging $p_{i f}=0$ into $\Delta_{i}$, we get $p_{i s}=T_{H} / \rho\left(1-\mu_{i}\right)$. In brief, the optimal payments after a signal $s_{i}$ are

$$
p_{i f}=0, \quad p_{i s}=\frac{T_{H}}{\rho\left(1-\mu_{i}\right)} .
$$

It remains to check that this contract indeed satisfies the constraint $(I R)$. The expert's expected profits and the consumer's expected surplus under the contact are

$$
\Pi^{s}\left(T_{H}\right)=\left[\frac{\lambda \mu_{L}}{1-\mu_{L}}+\frac{(1-\lambda) \mu_{H}}{1-\mu_{H}}\right] T_{H}, \quad S_{H}^{s}=\rho L-\left[\frac{\lambda}{1-\mu_{L}}+\frac{1-\lambda}{1-\mu_{H}}\right] T_{H}
$$

where $\Pi^{s}\left(T_{H}\right)>0$ by (13). This proves that the contract in (89) for $i \in\{L, H\}$ is optimal. Q.E.D. 
Proof of Proposition 9: Define $\Delta_{L} \equiv \rho\left(p_{L s}-p_{L f}\right)$ and $\Delta_{H} \equiv \rho\left(p_{H s}-p_{H f}\right)$. Ignoring the constraint $(I R)$ for the moment, the consumer's problem is then reduced to

$$
\min _{\left\{\left(p_{L s}, p_{L f}\right),\left(p_{H s}, p_{H f}\right)\right\}} \lambda\left(p_{L f}+\mu_{L} \Delta_{L}\right)+(1-\lambda)\left(p_{H f}+\Delta_{H}\right)
$$

subject to

$$
\begin{aligned}
& \left(1-\mu_{L}\right) \Delta_{L} \leq T_{H}, \\
& \left(1-\mu_{H}\right) \Delta_{H} \geq T_{H}, \\
& p_{L f} \geq 0, \quad p_{H f} \geq 0, \\
& p_{L f}+\Delta_{L} \geq 0, \quad p_{H f}+\Delta_{H} \geq 0,
\end{aligned}
$$

Obviously, it follows immediately from the first line of $(L L)$ that $p_{L f}=p_{H f}=0$ as the other constraints impose no further restriction and the total payments are increasing in $p_{L f}$ and $p_{H f}$. By substituting $p_{L f}=p_{H f}=0$ into the second line of $(L L)$ and combining it with $\left(I C_{L}\right)$ and $\left(I C_{H}\right)$, we get

$$
\begin{aligned}
& 0 \leq \Delta_{L} \leq \frac{T_{H}}{1-\mu_{L}} \\
& \Delta_{H} \geq \frac{T_{H}}{1-\mu_{H}} .
\end{aligned}
$$

Note that the total payments are strictly increasing both in $\Delta_{L}$ and in $\Delta_{H}$. Thus setting $\Delta_{L}=0$ and $\Delta_{H}=T_{H} /\left(1-\mu_{H}\right)$ is optimal. By definition $\Delta_{L}$ and $\Delta_{H}$ we obtain the payments

$$
p_{L s}=p_{L f}=p_{H f}=0, \quad p_{H s}=\frac{T_{H}}{\rho\left(1-\mu_{H}\right)} .
$$

Under the contract, the profits of the agent $t$ and the consumer's expected surplus are

$$
\Pi^{s}\left(T_{S}\right)=(1-\lambda) \frac{\mu_{H} T_{H}}{1-\mu_{H}}, \quad S_{S}^{s}=\left(\lambda \mu_{L}+1-\lambda\right) \rho L-\frac{1-\lambda}{1-\mu_{H}} T_{H} .
$$

Note that $\Pi^{s}\left(T_{S}\right)>0$ by (13), which implies that this contract indeed satisfies the constraint $(I R)$. Therefore, the contract in (94) is optimal.

Q.E.D.

Proof of Proposition 10: Note that $S_{L S}^{s}(q)$ is increasing in $q$ as well because

$$
\frac{\partial S_{L S}^{s}(q)}{\partial q}=\left(\frac{1-\mu_{H}+q \mu_{H}^{\prime}}{\left(1-\mu_{H}\right)^{2}}+1\right)(1-\sigma) T_{H}+\sigma\left(\rho L-T_{H}\right)>0
$$

by $\mu_{H}^{\prime} \equiv \partial \mu_{H} / \partial q>0$ and by (13).

In addition, as $S_{L S}^{s}(0)=\sigma\left(T_{H}-\rho L\right)<0$ and $S_{L S}^{s}(q) \rightarrow+\infty$ for $q \rightarrow 1$, by continuity there exists a unique $q_{L}^{s} \in(0,1)$ such that

$$
S_{L S}^{s}\left(q_{L}^{s}\right)=0
$$

Note that

$$
S_{L S}^{s}\left(q_{L}\right)=\frac{q_{L}(1-\sigma) T_{H}}{1-\mu_{H}}>S_{L S}^{s}\left(q_{L}^{s}\right) \equiv 0>S_{L S}^{s}\left(\hat{q}_{L}\right)=-\frac{\hat{q}_{L} \sigma T_{H}}{1-\mu_{H}} .
$$


Thus,

$$
q_{L}>q_{L}^{s}>\hat{q}_{L}
$$

as $\partial S_{L S}^{s}(q) / \partial q>0$.

On the other hand, $S_{H S}^{s}(q)$ is decreasing in $q$ because

$$
\frac{\partial S_{H S}^{s}(q)}{\partial q}=(\sigma-1)\left(\rho L-T_{H}\right)-\sigma\left(\frac{1-\mu_{H}+q \mu_{L}^{\prime}}{\left(1-\mu_{L}\right)^{2}}+1\right) T_{H}<0 .
$$

Furthermore, since $S_{H S}^{s}(0)=(1-\sigma)\left(\rho L-T_{H}\right)>0$ and $S_{H S}^{s}(1) \rightarrow-\infty$ with for $q \rightarrow 1$, there exists an unique $q_{H}^{s} \in(0,1)$ such that

$$
S_{H S}\left(q_{H}^{s}\right)=0
$$

Note that

$$
\begin{aligned}
S_{H S}^{s}\left(q_{H}\right) & =-\frac{\lambda \mu_{L}}{1-\mu_{L}} T_{H}<S_{H S}^{s}\left(q_{H}^{s}\right) \equiv 0, \\
S_{H S}^{s}\left(\hat{q}_{H}\right) & =(1-\lambda)\left(\frac{1}{1-\mu_{L}}-\frac{1}{1-\mu_{H}}\right) T_{H}-\frac{\lambda \mu_{L} T_{H}}{1-\mu_{H}} \\
& =\frac{\hat{q}_{H}\left(\sigma^{2}+\sigma-1\right)}{(1-\sigma)\left(1-\mu_{H}\right)} .
\end{aligned}
$$

Therefore, if $\gamma-1=(\sqrt{5}-1) / 2<\sigma<1$,

$$
S_{H S}^{s}\left(\hat{q}_{H}\right)>S_{H S}^{s}\left(q_{H}^{s}\right) \equiv 0 .
$$

This yields

$$
\hat{q}_{H}<q_{H}^{s}<q_{H}
$$

as $\partial S_{H S}^{s}(q) / \partial q<0$. Otherwise, if $1 / 2<\sigma<(\sqrt{5}-1) / 2=\gamma-1$, we have

$$
q_{H}^{s}<\hat{q}_{H}<q_{H}
$$

Q.E.D. 


\section{References}

AKerlof, G. (1970): "The Market for Lemons: Quality Uncertainty and the Market Mechanism," The Quarterly Journal of Economics, 89, 488-500.

Alger, I., AND F. SAlaniÉ (2006): "A Theory of Fraud and Over-Consumption in Experts Markets," Journal of Economics \& Management Strategy, 15, 853-81.

Balafoutas, L., A. Beck, R. Kerschbamer, and M. Sutter (2013): "What Drives Taxi Drivers? A Field Experiment on Fraud in a Market for Credence Goods," Review of Economic Studies, 80, 876-891.

BerWick, D., ANd A. Hackbarth (2012): "Eliminating Waste in US Health Care," JAMA, 307(14), 1513-1516.

Bester, H., And M. DAhm (2017): "Credence Goods, Costly Diagnosis, and Subjective Evaluation," Economic Journal (forthcoming), DOI: 10.1111/ecoj.12472.

Bonroy, O., S. Lemarié, AND J. TROpÉANo (2013): "Credence goods, Experts and Risk Aversion," Economics Letters, 120, 464-67.

DARbY, M., AND E. KARNi (1973): "Free Competition and the Optimal Amount of Fraud," Journal of Law and Economics, 16, 67-88.

DEMSKI, J. S., AND D. E. M. SAPPINGTON (1987): "Delegated Expertise," Journal of Accounting Research, 25(1), 68-89.

Dulleck, U., J. GonG, AND J. LI (2015): "Contracting for Infrastructure Projects as Credence Goods," Journal of Public Economic Theory, 17, 328-345.

Dulleck, U., And R. Kerschbamer (2006): "On Doctors, Mechanics, and Computer Specialists: The Economics of Credence Goods," Journal of Economic Literature, XLIV, 5-42.

(2009): "Experts vs. Discounters: Consumer Free-riding and Experts Withholding Advice in Markets for Credence Goods," International Journal of Industrial Organization, $27,15-23$.

Emons, W. (1997): "Credence Goods and Fraudulent Experts," Rand Journal of Economics, 28, 107-19.

_ (2001): "Credence Goods Monopolists," International Journal of Industrial Organization, 19, 375-89.

FonG, Y. (2005): "When Do Experts Cheat and Whom Do They Target," Rand Journal of Economics, 28, 113-30.

FONG, Y., T. LIU, AND D. J. WRIGHT (2014): "On the Role of Verifiability and Commitment in Credence Goods Market," International Journal of Industrial Organization, 37, 118-129. 
Gabszewicz, J. J., And J. Resende (2012): "Differentiated Credence Goods and Price Competition," Information Economics and Policy, 24(3-4), 277 - 287.

Glazer, J., And T. McGuire (1996): "Price Contracts and Referrals in Markets for Services," Working Paper No. 10/96, Institute of Business Research, Tel Aviv University.

Hilger, N. G. (2016): “Why Don't People Trust Experts?,” Journal of Law and Economics, 59, 293-311.

HYNDMAN, K., AND S. OzerTuRK (2011): "Consumer Information in a Market for Expert Services," Journal of Economic Behavior \& Organization, 80(3), 628- 640.

LIU, T. (2011): "Credence Goods Markets with Conscientious and Selfish Experts," International Economic Review, 52, 227-244.

Myerson, R. B. (1979): "Incentive Compatibility and the Bargaining Problem," Econometrica, 47, 61-73.

— (1982): "Optimal Coordination Mechanisms in Generalized Principal-Agent Problems," Journal of Mathematical Economics, 10, 67-81.

Nelson, P. (1970): "Information and Consumer Behavior," Journal of Political Economy, 78(2), 311-329.

Pesendorfer, W., And A. Wolinsky (2003): "Second Opinions and Price Competition: Inefficiency in the Market for Expert Advice," Review of Economic Studies, 70, 417-437.

Pitchik, C., And A. Schotter (1987): "Honesty in a Model of Strategic Information Transmission," American Economic Review, 77, 1032-36.

Richardson, H. (1999): "The Credence Good Problem and the Organization of Health Care Markets," TexasA \&M University. Mimeo.

SAPpington, D. (1983): "Limited Liability Contracts between Principal and Agent," Journal of Economic Theory, 29(1), 1-21.

SCHNEIDER, H. (2012): "Agency problems and Reputation in Expert Services: Evidence from Auto Repair," Journal of Industrial Economics, 60(3), 406-33.

SÜLZLE, K., AND A. WAMBACH (2005): "Insurance in a Market for Credence Goods,” Journal of Risk and Insurance, 72, 159-176.

TAYlOR, C. (1995): “The Economics of Breakdowns, Checkups, and Cures," Journal of Political Economy, 103, 53-74.

Thambisetty, S. (2007): "Patents as Credence Goods," Oxford Journal of Legal Studies, 27(4), 707-740.

Wolinsky, A. (1993): “Competition in a Market for Informed Experts' Services," Rand Journal of Economics, 24, 380-398. 


\section{Diskussionsbeiträge - Fachbereich Wirtschaftswissenschaft - Freie Universität Berlin Discussion Paper - School of Business and Economics - Freie Universität Berlin}

2017 erschienen:

2017/1 ARONSSON, Thomas und Ronnie SCHÖB

Habit Formation and the Pareto-Efficient Provision of Public Goods

Economics

2017/2 VOGT, Charlotte; Martin GERSCH und Cordelia GERTZ

Governance in integrierten, IT-unterstützten Versorgungskonzepten im Gesundheitswesen : eine Analyse aktueller sowie zukünftig möglicher Governancestrukturen und -mechanismen

Wirtschaftsinformatik

2017/3 VOGT, Charlotte; Martin GERSCH und Hanni KOCH

Geschäftsmodelle und Wertschöpfungsarchitekturen intersektoraler, IT-unterstützter Versorgungskonzepte im Gesundheitswesen Wirtschaftsinformatik

2017/4 DOMBI, Akos und Theocharis GRIGORIADIS

Ancestry, Diversity \& Finance : Evidence from Transition Economies Economics

2017/5 SCHREIBER, Sven

Weather Adjustment of Economic Output

Economics

2017/6 NACHTIGALL, Daniel

Prices versus Quantities: The Impact of Fracking on the Choice of Climate Policy Instruments in the Presence of OPEC

Economics

2017/7 STOCKHAUSEN, Maximilian

The Distribution of Economic Resources to Children in Germany Economics

2017/8 HETSCHKO, Clemens; Louisa von REUMONT und Ronnie SCHÖB Embedding as a Pitfall for Survey-Based Welfare Indicators: Evidence from an Experiment

Economics

2017/9 GAENTZSCH, Anja

Do Conditional Cash Transfers (CCT) Raise Educational Attainment? A Case Study of Juntos in Peru

Economics 
2017/10 BACH, Stefan; Martin BEZNOSKA und Viktor STEINER

An Integrated Micro Data Base for Tax Analysis in Germany

Economics

2017/11 NEUGEBAUER, Martin und Felix WEISS

Does a Bachelor's Degree pay off? Labor Market Outcomes of Academic versus Vocational Education after Bologna

Economics

2017/12 HACHULA, Michael und Dieter NAUTZ

The Dynamic Impact of Macroeconomic News on Long-Term Inflation

Expectations

Economics

2017/13 CORNEO, Giacomo

Ein Staatsfonds, der eine soziale Dividende finanziert

Economics

2017/14 GERSCH, Martin; Cordelia GERTZ und Charlotte VOGT

Leistungsangebote in integrierten, IT-unterstützten Versorgungskonzepten:

eine Konzeption (re-) konfigurierbarer Servicemodule im Gesundheitswesen

Wirtschaftsinformatik

2017/15 KREUTZMANN, Ann-Kristin; Sören PANNIER; Natalia ROJAS-PERILLA; Timo SCHMID; Matthias TEMPL und Nikos TZAVIDIS

The R Package emdi for Estimating and Mapping

Regionally Disaggregated Indicators

Economics

2017/16 VOGT, Charlotte; Cordelia GERTZ und Martin GERSCH

Ökonomische Evaluation eines integrierten, IT-unterstützten

Versorgungskonzepts im Gesundheitswesen: eine ökonomische Analyse von

E-Health-unterstützten Versorgungsprozessen

Wirtschaftsinformatik

2017/17 GASTEIGER, Emanuel und Klaus PRETTNER

A Note on Automation, Stagnation, and the Implications of a Robot Tax

Economics

2017/18 HAASE, Michaela

The Changing Basis of Economic Responsibility: zur Bedeutung und

Rezeption von John Maurice Clarks Artikel zur ökonomischen Verantwortung Marketing

2017/19 FOSSEN, Frank M.; Ray REES; Davud ROSTAM-AFSCHAR und Viktor STEINER

How Do Entrepreneurial Portfolios Respond to Income Taxation?

Economics 
2017/20 NEIDHÖFER, Guido; Joaquín SERRANO und Leonardo GASPARINI

Educational Inequality and Intergenerational Mobility in Latin America: A New Database

Economics

2017/21 SCHMITZ, Sebastian: The Effects of Germany's New Minimum Wage on Employment and Welfare Dependency

Economics

2017/22 WALTER, Paul; Marcus GROß, Timo SCHMID und Nikos TZAVIDIS:

Estimation of Linear and Non-Linear Indicators using Interval Censored Income Data

Economics

2017/23 WAGNER, Julia: Zinsbereinigte Besteuerung und Verlustvortrag : eine Mikrosimulation für deutsche Kapitalgesellschaften

FACTS

2017/24 CRUSIUS, Tobias L. und Marten von WERDER

The Affluency to Quit: How Inheritances Affect Retirement Plannings

Economics

2017/25 ALHO, Juha; Gerrit MÜLLER, Verena PFLIEGER und Ulrich RENDTEL The Fade Away of an Initial Bias in Longitudinal Surveys

Economics

2017/26 JESSEN, Robin; Maria METZING und Davud ROSTAM-AFSCHAR

Optimal Taxation Under Different Concepts of Justness

Economics

2017/27 LEONE, Tharcisio

The gender gap on intergenerational mobility: Evidence of educational persistence in Brazil

Economics

2017/28 HAAN, Peter; Daniel KEMPTNER und Holger Lüthen

The rising longevity gap by lifetime earnings - distributional implications for the pension system

Economics

2017/29 PREUSS, Malte und Juliane HENNECKE

Biased by Success and Failure: How Unemployment Shapes Stated Locus of Control

Economics

2017/30 ROJAS-PERILLA, Natalia; Sören PANNIER; Timo SCHMID und Nikos

TZAVIDIS

Data-Driven transformations in small area estimation

Economics 